This document is the Accepted Manuscript version of a Published Work that appeared in final form in Soft Matter, 2009, 5, 2614-2624, copyright (C) Royal Society of Chemistry after peer review and technical editing by the publisher. To access the final edited and published work see http://pubs.rsc.org/en/Content/ArticleLanding/2009/SM/b819189a

\title{
Static and dynamic structural probing of swollen Polyacrylamide ferrogels
}

\author{
J.A. Galicia ${ }^{1,2}$, F.Cousin ${ }^{3}$, E. Dubois ${ }^{1}$, O. Sandre ${ }^{1}$, V. Cabuil ${ }^{1}$, R. Perzynski ${ }^{1, *}$ \\ ${ }^{1}$ Université Pierre et Marie Curie - PECSA - UMR 7195 CNRS - UPMC - ESPCI, case 51, 4 Place Jussieu, 75252 Paris cedex 05 - France \\ ${ }^{2}$ Fac. de Ing. Quimica, Benemerita Universidad Autonoma de Puebla, Edif 147/102 Ciudad Universitaria,18 sur y San Claudio, Puebla, Pue. Mexico \\ ${ }^{3}$ Laboratoire Léon Brillouin, UMR 12 CNRS-CEA, CE-Saclay, 91191 Gif-sur-Yvette - France
}

Received $30^{\text {th }}$ October 2008, Accepted $3^{\text {rd }}$ April 2009

First published on the web as an Advance Article on the web 15th May 2009

DOI: $10.1039 / b 819189 a$

* corresponding author, e-mail : regine.perzynski@upmc.fr

The local structure of hybrid ferrogels resulting from the incorporation of $\gamma-\mathrm{Fe}_{2} \mathrm{O}_{3}$ nanoparticles in a polyacrylamide polymeric network is probed from a static and a dynamic point of view, in various synthesis conditions and at swelling equilibrium. Relaxation of magneto-optical birefringence and Small Angle Neutron Scattering measurements show an adsorption of the nanoparticles onto the polymer ascribed to H-bonds. These measurements allow sorting out the conditions leading either - to a homogeneous scaffold reinforced by the nanoparticles or - to a nano-structured composite with a 2D nanoparticle decoration on the walls of percolating pockets, initially filled by the ferrofluid during the synthesis and leaking out during the swelling. Two parameters rule the structure and the properties of these ferrogels : - the osmotic pressure ratio $\mathrm{R}_{\Pi}$ (ratio of the osmotic pressure of the initial ferrofluid to that of the hydrogel) and the steric parameter $R_{S}$ (ratio of the nanoparticles volume to the mesh volume of the polymeric hydrogel).

\section{Introduction}

Ferrogels are hybrid materials resulting from the mixing of either micrometric or nanometric magnetic particles with polymeric chains. Such composites are usually developed for their magneto-elastic properties. ${ }^{1,2}$ The particles, frequently aggregated, reinforce the polymeric matrix mechanically., ${ }^{3,45}$ More generally, nanocomposites successfully combining polymers and magnetic nanoparticles result from the synthesis of multifunctional 3D architectures rationally assembled at the nanoscale. We disperse here magnetic nanoparticles of controlled size and coating inside polyacrylamide (PAM) hydrogels. PAM is well known for its swelling properties in aqueous media, it is often used as super-absorbing microbeads and in biochemistry. ${ }^{6}$ Our aim here is to monitor the local structure of our ferrogel by using the nanoparticles both as fillers and as probes, an aspect quite rarely reported in literature $^{7}$ even though polymers are widely used to template different complex nanoparticles assemblies. ${ }^{8}$ By tuning the physico-chemical parameters of the system, we are able to control for example - the volume fraction of internalized nanoparticles and their release, - the swelling of the ferrogel, the local homogeneity of the hybrid material, - the proportion of internalized nanoparticles free to rotate and those blocked in rotation. The control of the fraction of the internalized particles at equilibrium after swelling is specifically important for the mechanical properties, as it has been shown that the Young modulus of the ferrogel strongly depends on this fraction. ${ }^{2,9}$

The synthesis ${ }^{9}$ is performed by polymerizing acrylamide (AM) monomers in an aqueous suspension of maghemite $(\gamma$ $\mathrm{Fe}_{2} \mathrm{O}_{3}$ ) nanoparticles in the presence of the cross-linking agent (BAM). The ratio $\mathrm{CL}=\mathrm{BAM} / \mathrm{AM}$ allows a tuning of the meshsize of the polymeric network. The ferrogels are then let to reach their equilibrium inside a swelling bath because they are out-of-equilibrium materials in the synthesis conditions.
To probe our ferrogels from both a static and a dynamic point of view, we take advantage of the physical properties of the dispersed nanoparticles. We study their local organization inside the medium by Small Angle Neutron Scattering (SANS) $)^{10,11}$, taking benefit from the possibility to match the scattering length density of PAM in mixture of $\mathrm{H}_{2} \mathrm{O}$ and $\mathrm{D}_{2} \mathrm{O}$. Being magnetic monodomains and also optically uniaxial, the nanoparticles give optical responses to magnetic solicitations. We perform a nano-mechanical probing of the hybrid system by a magneto-optical birefringence method, which is specific to ferrofluids. ${ }^{12,13,14}$ We are thus able to obtain a local, structural and rheological information on the hybrid system at the length scale of the nanoparticles.

In the following, we first discuss the parameters of the initial hydrogels and ferrofluids that can influence the local structure of ferrogels. After exposing our choices for the current study, we present the synthesis and the physicochemical properties of the ferrogels. Their structural and magneto-relaxational properties are then presented and discussed in terms of homogeneity/inhomogeneity of the complex magnetic medium.

\section{Choice of components for designing ferrogels with versatile structures}

If one wants to define a reproducible procedure for synthesizing ferrogels, versatile enough to tune the structure of the ferrogel from very homogenous to heterogeneous, the first step is to find physico-chemical conditions for the synthesis at which the individual components of the system are homogenous prior to mixing.

This implies to choose dispersions of magnetic nanoparticles with an interparticle potential dominated by repulsion in order to avoid any kind of aggregation of the nanoparticles before ferrogel synthesis. This has led us to work with ionic 
This document is the Accepted Manuscript version of a Published Work that appeared in final form in Soft Matter, 2009, 5,

2614-2624, copyright (C) Royal Society of Chemistry after peer review and technical editing by the publisher. To access the final edited and published work see http://pubs.rsc.org/en/Content/ArticleLanding/2009/SM/b819189a

dispersions of magnetic nanoparticles based on maghemite $(\gamma$ $\mathrm{Fe}_{2} \mathrm{O}_{3}$ ), of typical size $10 \mathrm{~nm}$, coated by citrate species that provide them with a negative charge in water at neutral $\mathrm{pH}^{15}$ For a citrate salt concentration $\left[\mathrm{Na}_{3} \mathrm{Cit}\right]$ lying between $2.10^{-3}$ mol. $L^{-1}$ and $10^{-2}$ mol. $L^{-1}$, the electrostatic repulsion dominates the interparticle attraction and the phase behavior is well described by the usual fluid-solid phase diagram of a repulsive system, even when a magnetic field is applied. ${ }^{16}$ We have decided to fix the $\mathrm{pH}$ to 7.2 for all the experiments described in the paper and the citrate salt concentration [Na $\left.\mathrm{Na}_{3} \mathrm{Cit}\right]$ to $8.10^{-3}$ mol. $L^{-1}$

For the hydrogel, it means to work in a range of cross-linking CL where single hydrogels have both (i) good mechanical performances and (ii) an homogeneous structure. We have thus decided to work in a range of CL lying between $0.5 \%$ and $2 \%$ because the gels are too soft to be manipulated below CL = $0.5 \%$ and PAM hydrogels are known to present local heterogeneities of cross-linkers ${ }^{17,18}$ above CL $=2 \%$. For these cross-linking ratios $0.5 \% \leq \mathrm{CL} \leq 2 \%$, the mesh-size $\xi_{\text {synth }}$ of the polymeric hydrogel after the synthesis ranges between 11 and $17 \mathrm{~nm}$ (see Table SI.1 of Supporting Informations (SI)).

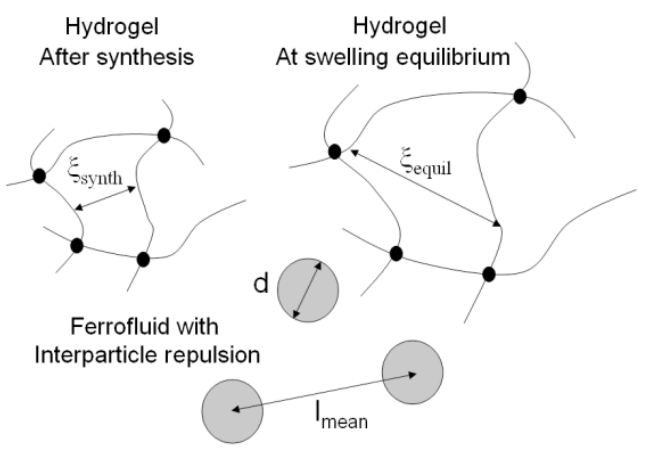

Figure 1 - Characteristic sizes of the individual components which are mixed together to form the ferrogel - Hydrogel : mesh size after synthesis $\xi_{\text {synth }}$, (upper left) and at swelling equilibrium $\xi_{\text {equil }}$ (upper right). Ferrofluid : characteristic diameter $\mathrm{d}$ of the magnetic nanoparticle and interparticle distance $l_{\text {mean }}$ (down).

Two parameters may play a decisive role on the structure and on the properties of the ferrogel when the components are mixed:

- the osmotic parameter $R_{\Pi}$, ratio of the osmotic pressure of the suspension of magnetic nanoparticles and that of the hydrogel, - the steric parameter $\mathrm{R}_{\mathrm{S}}$, ratio of the characteristic volumes of these components.

Both $R_{\Pi}$ and $R_{S}$ depend on the nanoparticle characteristics and on the choice of cross-linking ratio, $R_{\Pi}$ being also dependent on the volume fraction $\Phi_{\text {synth }}$ of the initial ferrofluid (which fixes the initial interparticle distance $l_{\text {mean }}$ ).

The osmotic parameter $\mathrm{R}_{\Pi}=\Pi^{F F} / \Pi_{s w l}^{H G}$ is the ratio of the osmotic pressures that characterizes the two components of the mixture in the initial conditions (see SI). If the osmotic pressure $\Pi^{F F}$ of mixing of the nanoparticles with the solvent in the pure ferrofluid is much larger than the initial swelling pressure $\Pi_{s w l}^{H G}$ of the pure hydrogel, it may be impossible to incorporate nanoparticles in the gels.

The steric parameter $\mathrm{R}_{\mathrm{S}}=d^{3} / \xi_{\text {synth }}^{3}$ is the ratio between the nanoparticle characteristic volume and the mesh volume $\xi_{\text {synth }}^{3}$ of the polymeric hydrogel, supposed homogeneous, immediately after synthesis. If $R_{S}$ is too large, the presence of the nanoparticles may prevent to form a homogeneous gel.

Prior to mix any components, it is thus important to choose carefully the mesh size $\xi_{\text {synth }}$ of the hydrogel after synthesis as well as its swelling pressure $\Pi_{\text {swell }}^{H G}$, together with the nanoparticle size and the osmotic pressure $\Pi^{F F}$ of the ferrofluid dispersion. The way in which all these details are obtained, is given in SI.

\section{Ferrogels: synthesis and characterizations}

a) Synthesis

The polymerization of the ferrogels is performed by a free radicalar polymerization at a given CL, in the aqueous dispersion of magnetic nanoparticles, at a given $\Phi_{\text {synth }}$ tuned from $1 \%$ to $7 \%$ and at $\left[\mathrm{Na}_{3} \mathrm{Cit}\right]=8 \cdot 10^{-3} \mathrm{~mol} . \mathrm{L}^{-1}$. All chemicals, including the monomer $\mathrm{AM}$ and the cross-linker BAM are purchased from Sigma-Aldrich. The reactional mixture of global volume $3 \mathrm{~mL}$, contains acrylamide monomers $\mathrm{AM}$ (concentration 0.5 mol.L ${ }^{-1}$ ), cross-linking agent N,N'methylen-bis-acrylamide (BAM) and the initiator (Ammonium persulfate at a concentration of $4.10^{-4} \mathrm{~mol} . \mathrm{L}^{-1}$. Solutions are degassed under nitrogen for 5 minutes to eliminate oxygen, which would hamper the polymerization. The solutions are maintained at $70^{\circ} \mathrm{C}$ during two hours to achieve the completion of the reaction. The ferrogel appears as a red/orange material because of the trapped $\gamma-\mathrm{Fe}_{2} \mathrm{O}_{3}$ particles.

At the end of the synthesis, the ferrogels are molden as cylinders of polymeric concentration $\varphi_{A M}^{\text {synth }} \sim 3 \%$. They are carefully removed from their vessel and then swollen in an aqueous solution at $\left[\mathrm{Na}_{3} \mathrm{Cit}\right]=0.008 \mathrm{~mol} \cdot \mathrm{L}^{-1}$. The swelling equilibrium of the ferrogels is reached in three weeks during which the swelling bath is changed several times. The particles, which are not trapped, are released in the swelling bath. ${ }^{9}$ Both phenomena of solvent swelling and particle release change the volume fraction of particles $\Phi_{\text {equil }}$ inside the gel which is then smaller than $\Phi_{\text {synth. }}$.

b) Conditions chosen for the synthesis of ferrogels

The determination of the characteristic spatial scales of the individual components (table 1 and 2 of SI) enable us to choose conditions for synthesis of ferrogels where $R_{S}$ is higher or lower than 1 and $R_{\Pi}$ is varied on a large range. Two different batches of ferrofluids with slightly different typical size of nanoparticles (FFA with $\mathrm{d}=12 \mathrm{~nm}$ and FFB with $\mathrm{d}=11 \mathrm{~nm}$ ) are used here. Ferrogels prepared with nanoparticles FFA (resp. FFB) are denoted FGA (resp. FGB).

For $\mathrm{CL}=0.5 \%$ and $1 \%, \mathrm{R}_{\mathrm{S}}$ is less than 1 for both kinds of nanoparticles FFA and FFB. The case of FFA with CL $=1 \%$ has been already considered in ${ }^{9}$. In the following we thus limit ourselves to the synthesis of ferrogels at CL $=0.5 \%$ with FFA to picture ferrogels with $\mathrm{R}_{\mathrm{S}}<1$ and we compare the results with the ones from ${ }^{9}$. To picture ferrogels with $R_{S} \geq 1$ we consider both FFA and FFB for CL $=2 \%$ as $R_{S}>1$ for FFA and $\mathrm{R}_{\mathrm{S}} \sim 1$ for FFB. Table 1 summarizes the values of $\mathrm{R}_{\mathrm{S}}$ in the various conditions of ferrogel synthesis.

\begin{tabular}{|c|c|c|c|}
\hline $\begin{array}{c}\text { FGA } \\
\mathrm{CL}=0.5 \%\end{array}$ & $\begin{array}{c}\text { FGA } \\
\mathrm{CL}=1 \%\end{array}$ & $\begin{array}{c}\text { FGB } \\
\mathrm{CL}=2 \%\end{array}$ & $\begin{array}{c}\text { FGA } \\
\mathrm{CL}=2 \%\end{array}$ \\
\hline $\mathrm{R}_{\mathrm{S}}=0.35$ & $\mathrm{R}_{\mathrm{S}}=0.7$ & $\mathrm{R}_{\mathrm{S}}=1$ & $\mathrm{R}_{\mathrm{S}}=1.3$ \\
\hline
\end{tabular}

Table 1 - Steric parameter $\boldsymbol{R}_{S}$ values for the various ferrogels

Figure 2 compares the swelling pressure $\Pi_{s w l}^{H G}$ of the hydrogels to the equation of state $\Pi^{F F}(\Phi)$ of the ferrofluid dispersions at $\left[\mathrm{Na}_{3} \mathrm{Cit}\right]=8.10^{-3} \mathrm{~mol} . \mathrm{L}^{-1}$ as given by the Carnahan-Starling formalism - equ. (SI.7). It allows us, by using osmotic stress, to 
This document is the Accepted Manuscript version of a Published Work that appeared in final form in Soft Matter, 2009, 5,

2614-2624, copyright (C) Royal Society of Chemistry after peer review and technical editing by the publisher. To access the final edited and published work see http://pubs.rsc.org/en/Content/ArticleLanding/2009/SM/b819189a

adjust precisely the osmotic pressure $\Pi^{F F}$ inside the ferrofluid used for preparing ferrogels and thus also the volume fraction of particles $\Phi_{\text {synth }}$. We prepare ferrogels in a large range of known osmotic parameter $\mathrm{R}_{\Pi}$ varying from $\mathrm{R}_{\Pi}<<1$ to $\mathrm{R}_{\Pi} \sim 1$. Let us point out that it has not been possible here to synthesize macroscopic homogeneous ferrogels FGA at $\mathrm{CL}=2 \%$, that would correspond to $\mathrm{R}_{\Pi} \geq 1$.

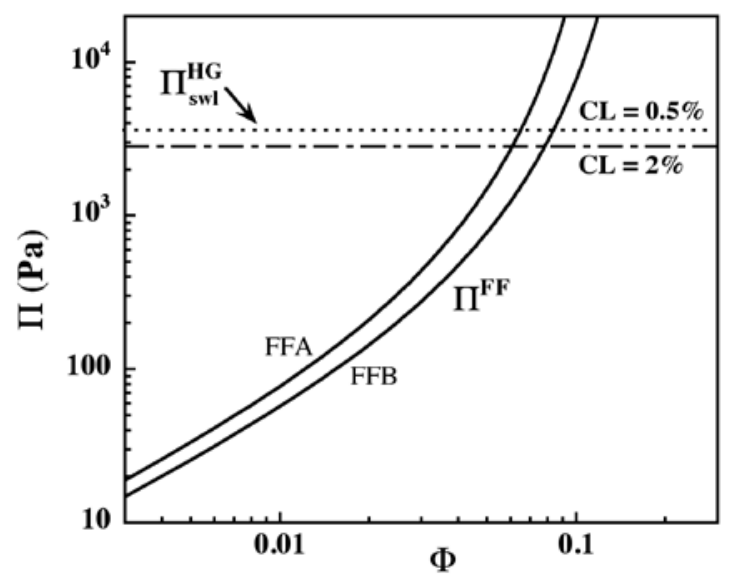

Figure 2 - Hydrogels : Initial swelling pressure $\Pi_{s w l}^{H G}$ at $C L=0.5 \%$ (horizontal dotted line) and at CL $=2 \%$ (horizontal dashed-dotted line (Table SI.1 of SI). Ferrofluids : Carnahan Starling adjustments of the osmotic pressure $\Pi^{F F}(\Phi)$ of nanoparticles FFA and FFB following equ. (SI.7) - full lines (parameters : see table SI.2).

\section{c) Structural and rotational probing of the ferrogels}

We probe the structural organization of nanoparticles in the ferrogels on a local scale by SANS. The experiments are performed in LLB (Saclay, France) on the PAXY spectrometer. We play on the neutronic contrast of the solvent to match the polymer scattering. The solvent is a mixture $\mathrm{H}_{2} \mathrm{O} / \mathrm{D}_{2} \mathrm{O}(44 / 56$ vol\%). Its density of neutron scattering length is equal to that of the polymer, taking into account the exchange between hydrogen and deuterium of the two labile protons of the PAM repetition unit. In these conditions, the scattering coming from the nanoparticles is mainly of nuclear origin, with a contrast $\Delta \rho^{2}$ equal to $1.33 .10^{21} \mathrm{~cm}^{-4}$, the magnetic contribution being less than $7 \%$ of the nuclear one. ${ }^{19}$ The scattered intensity is :

$$
\frac{I(q, \Phi)}{\Phi}=\Delta \rho^{2} F(q) S(q, \Phi)
$$

where $F(q)$ is the form factor of the nanoparticles and $S(q, \Phi)$ the structure factor of the dispersed nanoparticles. The structure factor in swollen ferrogels at various volume fractions of nanoparticles are obtained by dividing the scattering of ferrogels by the form factor of the nanoparticles measured with pure and dilute ferrofluids (shown for FFB in Fig SI.2 of SI). The shape of the structure factor reflects the homogeneity/inhomogeneity of the nanoparticles dispersion and allows measuring the most probable value of the interparticle distance inside the complex medium.

A complementary probing of the rotational degree of freedom of the nanoparticles at the nanoscale, inside the medium where they are dispersed, is also realized. Each nanoparticle of maghemite bears a magnetic moment $\frac{U}{\sqrt{x}}$ and an optical anisotropy axis $\dot{\phi}$, which are linked together by the anisotropy energy. Under an applied field $\sqrt{\mathrm{H}}$, the magnetic

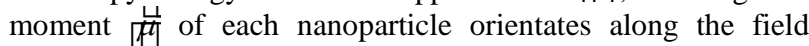
direction. If the nanoparticle is free to rotate in its carrier medium, this also rotates mechanically the core of the nanoparticle together with its optical axis. The alignment (even partial) of the optical axis $\ddot{\mid}$ of the nanoparticles imparts the system with a macroscopic optical birefringence $\Delta \mathrm{n}$. Comparing the birefringence induced in a ferrogel to that induced in a ferrofluid based on the same nanoparticles allows quantifying the proportion of nanoparticles free to rotate in the ferrogel and to characterize dynamically this rotation (see part 3 of SI). Two magneto-optical experiments are here performed to probe the rotation :

- A static measurement of the birefringence up to high fields (of the order of $10^{3} \mathrm{kA} / \mathrm{m}$ ) ${ }^{20}$; In high fields, the birefringence $\Delta \mathrm{n}$ saturates and is then proportional to the number of nanoparticles that can rotate within the sample (see SI - part 2).

- A dynamic measurement of the optical birefringence relaxation after a pulse of magnetic field with a small amplitude; this relaxation can be written as a stretched exponential decay

$$
I(t)=I_{0} \exp \left(-\left(t / \tau_{0}\right)^{\alpha}\right)
$$

where $\tau_{\mathrm{o}}$ is a characteristic time of rotation and the exponent $\alpha$ is related to the width of the distribution of relaxation times in the dispersion. In a pure and dilute ferrofluid, this relaxation reduces to the rotational diffusion of the nanoparticles.

More details on these experiments are given in part 2 of SI. They provide us with a powerful structural and rheological probing of the ferrogels on the nanoscale.

\section{d) Swelling ratio and particle release of the ferrogels}

These ferrogels present remarkable swelling properties in particular because they are out-of-equlibrium in the synthesis conditions. During the swelling process, some internalized nanoparticles may escape from the gel and are released in the swelling bath.

The swelling ratio of the ferrogels $G$ is here defined as :

$$
G=\frac{m_{\text {water }}}{m_{\text {dry }}^{\text {polymer }}}=\frac{m_{F G}-m_{\text {dry }}}{m_{\text {dry }}-m_{\text {part }}}
$$

It is measured as follows. The ferrogel is weighed $\left(m_{F G}\right)$ and dried at $70^{\circ} \mathrm{C}$ in order to obtain the mass of the dry ferrogel $\left(m_{\text {dry }}\right)$. The mass of $\gamma-\mathrm{Fe}_{2} \mathrm{O}_{3}\left(m_{\text {part }}\right)$ in the ferrogel is deduced from iron concentration determination by using either chemical titration or Atomic Absorption Spectroscopy.

Note that following expression (3), the swelling ratio of the ferrogel $G_{\text {synth }}$ at the end of the synthesis (thus before swelling) is already of the order of a few tens in our conditions. Hereafter we denote by $G_{\text {equil }}$ the swelling ratio of the ferrogel at swelling equilibrium.

In the pure hydrogels, the corresponding swelling ratios $G_{\text {synth }}^{H G}$ and $G_{\text {equil }}^{H G}$ are obtained by using exp. (3) with $\mathrm{m}_{\text {part }}=0$.

At swelling equilibrium, the ratio of nanoparticle release is calculated with:

$$
R=\frac{m_{\text {part }}^{\text {synt }}-m_{\text {part }}^{\text {equil }}}{m_{\text {part }}^{\text {synth }}}
$$

where $m_{\text {part }}^{\text {synt }}$ is the initial mass of $\gamma-\mathrm{Fe}_{2} \mathrm{O}_{3}$ introduced at the ferrogel synthesis. The release ratio $R$ is a characteristic parameter of the swollen ferrogels. It quantifies the nanoparticles ability to escape from the gel during swelling. The ferrogel always reaches an equilibrium state for which both $R$ and $G_{\text {equil }}$ remain constant. 
This document is the Accepted Manuscript version of a Published Work that appeared in final form in Soft Matter, 2009, 5, 2614-2624, copyright (C) Royal Society of Chemistry after peer review and technical editing by the publisher. To access the final edited and published work see http:// pubs.rsc.org/en/Content/ArticleLanding/2009/SM/b819189a

Figure 3 presents the swelling ratio $G_{\text {equil }}$ of the ferrogels as a function of $\Phi_{\text {synth }}$. For a comparison, the values obtained in ref ${ }^{9}$ are also plotted. The full line corresponds to the value $G_{\text {synth }}$ at the end of the synthesis before swelling. It is independent on the nanoparticle size distribution and varies from 25 to 27 in our range of $\Phi_{\text {synth }}$. Whatever $\Phi_{\text {synth }}$ and CL (except for $\Phi_{\text {synth }}=9 \%$ and $C L=1 \%$ ) the value $G_{\text {equil }}$ at the end of the swelling process is larger than $G_{\text {synth }}$. Moreover, whatever the volume fraction $\Phi_{\text {synth }}$ introduced, $G_{\text {equil }}$ is lower than the swelling ratio of the hydrogel $G_{\text {equil }}^{H G}$ at the same crosslinking ratio CL (see Fig. 3). The introduction of nanoparticles within the polymeric network strongly modifies the affinity of solvent for the polymer, expressing that the swelling pressure $\Pi_{\text {swell }}^{H G}$ of the ferrogel is weaker than the one of the corresponding hydrogel.

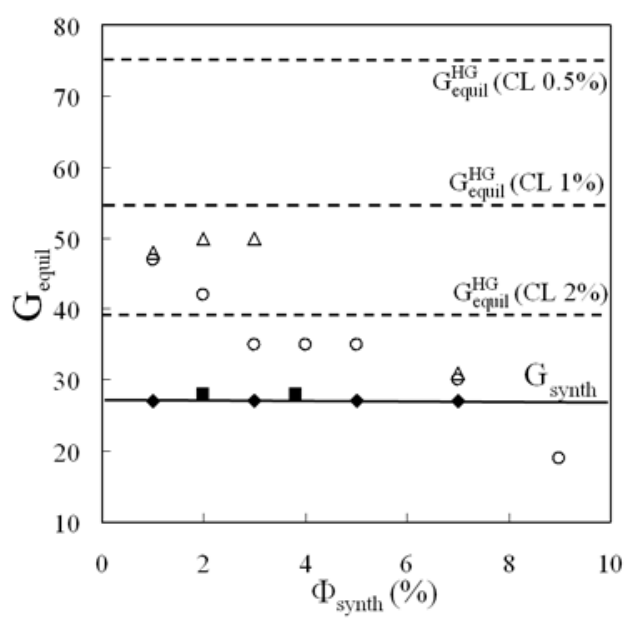

Figure 3 - Swelling ratio of swollen ferrogels $G_{\text {equil }}$ as a function of the initial volume fraction of nanoparticles $\Phi_{\text {synth }}$ : FGA with $\mathrm{CL}=0.5 \%$ (open triangles), $\mathrm{CL}=1 \%$ (open circles - data from $^{9}$ ) and $\mathrm{CL}=2 \%$ (solid squares); FGB - $\mathrm{CL}=2 \%$ (solid diamonds); The horizontal lines correspond to the swelling ratio $G_{\text {synth }}$ at the end of synthesis (full line) and to the swelling ratio of hydrogels at various CL (dashed lines).

As observed for the hydrogels, the swelling ratio $G_{\text {equil }}$ of ferrogels at constant $\Phi_{\text {synth }}$ decreases with increasing the crosslinking ratio. If $\Phi_{\text {synth }}$ increases at constant CL, $G_{\text {equil }}$ decreases except for $\mathrm{CL}=2 \%$, ferrogels then do not swell $\left(G_{\text {equil }}\right.$ is $\sim G_{\text {synth }}$ for FGA and FGB).

Figure 4 shows the evolution of the release ratio $R$ as a function of the initial volume fraction $\Phi_{\text {synth }}$ in the ferrogels.

For FGA ferrogels, the release ratio $\mathrm{R}$ increases with $\Phi_{\text {synth }}$ more or less linearly up to $\Phi_{\text {synth }}=4 \%\left(\mathrm{R}_{\Pi} \leq 0.2\right)$ and is independent on CL. For higher $\Phi_{\text {synth }}\left(\mathrm{R}_{\Pi}>0.2\right)$, it stops at a maximal value of the order of $45 \%$ for $C L=0.5 \%$ as it was observed in ref ${ }^{9}$ with FGA samples at CL $=1 \%$.

For FGB ferrogels at CL $=2 \%$, there is no release at low $\Phi_{\text {synth }}$ $\left(\mathrm{R}_{\Pi}<0.2\right)$ and a very large release ratio (about $\left.85 \%\right)$ at larger $\Phi_{\text {synth }}\left(R_{\Pi}>0.2\right)$. The difference of release ratios $\mathrm{R}$ between FGA and FGB samples at cross-linking ratio of $2 \%$ at low $\Phi_{\text {synth }}$ (both associated with $\mathrm{R}_{\Pi} \leq 0.2$ ) points out the important role of $R_{S}$. Both systems do not swell. But samples made with FFA $\left(R_{S}>1\right)$ release nanoparticles while samples made with FFB $\left(\mathrm{R}_{\mathrm{S}} \sim 1\right)$ do not.

The mass conservation implies:

$$
\Phi_{\text {equil }}=\Phi_{\text {synth }}(1-R) \frac{G_{\text {synth }}}{G_{\text {equil }}}
$$

This relation is well verified experimentally. The evolution of the volume fraction $\Phi_{\text {equil }}$ obtained at the end of the swelling process as a function of $\Phi_{\text {synth }}$ is presented in figure 5 for the different ferrogels.

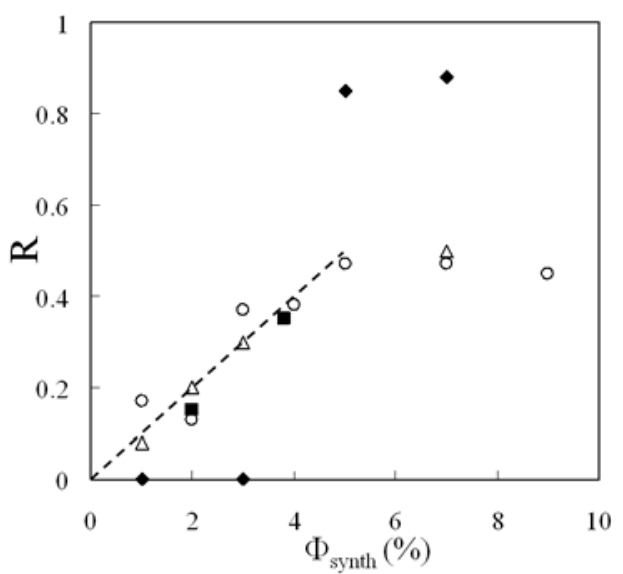

Figure 4 - Release ratio $\boldsymbol{R}$ versus $\boldsymbol{\Phi}_{\text {synth }}$. Same symbols as in Figure 3. The dashed line corresponds to R proportional to $\Phi_{\text {synth }}$ at $R_{\Pi} \leq 0.2$ for FGA.

At low cross-linking ratios (CL $=0.5 \%$ and $1 \%$ - FGA - open symbols) the results in Fig. 5 are roughly equivalent up to $\Phi_{\text {synth }}$ $=7 \%: \Phi_{\text {equil }}$ is an increasing function of $\Phi_{\text {synth }}$ with $\Phi_{\text {equil }}$, always smaller than $\Phi_{\text {synth. }}$. As the samples swell, the mesh size of the polymeric network initially larger than particles still increases allowing the nanoparticles release. On the contrary for $\mathrm{CL}=2 \%$ - FGB (solid diamonds), we have to distinguish two situations: $\Phi_{\text {synth }} \leq 3 \%\left(\mathrm{R}_{\Pi} \leq 0.2\right)$ and $\Phi_{\text {synth }}>3 \%\left(\mathrm{R}_{\Pi}>\right.$ 0.2 ). At low $\Phi_{\text {synth }}$ we obtain $\mathrm{R}=0$ and $G_{\text {equil }}=G_{\text {synth }}$ and thus $\Phi_{\text {equil }}=\Phi_{\text {synth }}$ (see Fig. 5). As the samples do not swell, the mesh size of the polymeric network remains always constant and close to the particle diameter. This indeed limits the translational diffusion of particles within the network and prevents release. For $\Phi_{\text {synth }}>3 \%$ the behavior drastically changes : R is large (Fig. 4) and $\Phi_{\text {synth }}$ decreases to a value of the order of $1 \%$ (Fig. 5).

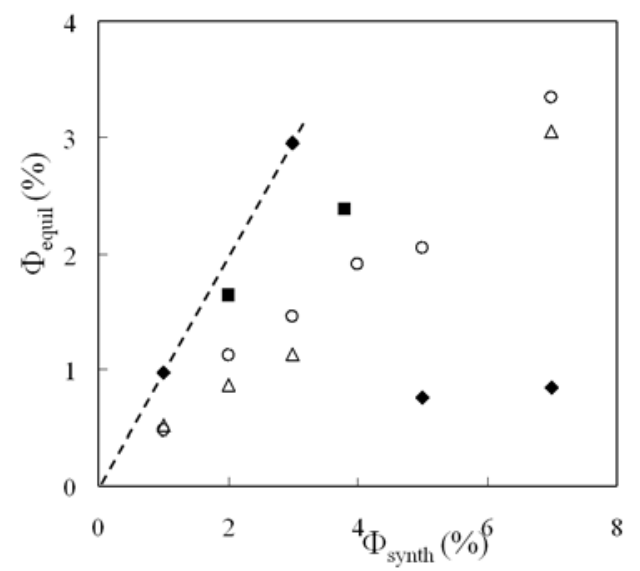

Figure 5 - Swollen ferrogels - Volume fraction of nanoparticles $\Phi_{\text {equil }}$ at swelling equilibrium versus the initial volume fraction $\Phi_{\text {synth }}$ for various ferrogels. Same symbols as in Figure 3. The dashed line corresponds to $\Phi_{\text {equil }}=\Phi_{\text {synth }}$.

\section{Ferrogels at $R_{s}<1$}

We study here the structure and properties of ferrogels before and after swelling in the limiting case where $\mathrm{R}_{\mathrm{S}}<1$. We choose thus to work with FGA samples at CL $=0.5 \%$ (see table 3 ) and we vary $R_{\Pi}$ in a large range. 
This document is the Accepted Manuscript version of a Published Work that appeared in final form in Soft Matter, 2009, 5, 2614-2624, copyright (C) Royal Society of Chemistry after peer review and technical editing by the publisher. To access the final edited and published work see http://pubs.rsc.org/en/Content/ArticleLanding/2009/SM/b819189a

\section{a) Rheological probing before swelling}

The nanoparticles able to rotate in the samples before swelling are probed dynamically by relaxation of birefringence for three different $\Phi_{\text {synth }}(1 \%, 3 \%$ and $7 \%)$. The experiments are performed on ferrogels instantaneously after the synthesis before they are placed in their swelling bath. The measurement of the relaxation time $\tau_{\mathrm{o}}$ and of the initial intensity $\mathrm{I}_{\mathrm{o}}$ enable to obtain the volume fraction of rotating nanoparticles in ferrogel $\Phi_{\text {synth }}^{\text {rot }}$ by comparison with the values of the pure ferrofluid (see principle in part 3 of SI). The values of $\Phi_{\text {synth }}^{\text {rot }} / \Phi_{\text {synth }}, \Phi_{\text {synth }}^{\text {rot }}$ and of $\Phi_{\text {synth }}^{\text {block }}\left(=\Phi_{\text {synth }}-\Phi_{\text {synth }}^{\text {rot }}\right)$ are collected in table 2 . Although the average mesh size of the polymeric network is superior to the particle diameter $\left(R_{S}=0.35\right)$, there is a large amount of nanoparticles which are not allowed to rotate within the ferrogel. This suggests an interaction with the polymeric network. However the proportion of rotating particles increases from $15 \%$ at $\Phi_{\text {synth }}=1 \%$ up to $64 \%$ at $\Phi_{\text {synth }}=7 \%$.

\begin{tabular}{|c|c|c|c|c|}
\hline$\Phi_{\text {synth }}(\%)$ & $\tau_{\mathrm{o}}(\mu \mathrm{S})$ & $\Phi_{\text {synth }}^{\text {rot }} / \Phi_{\text {synth }}$ & $\Phi_{\text {synth }}^{\text {rot }}(\%)$ & $\Phi_{\text {synth }}^{\text {block }}(\%)$ \\
\hline 1 & 3.2 & 0.15 & 0.15 & 0.85 \\
\hline 3 & 2.7 & 0.34 & 1.0 & 2 \\
\hline 7 & 1.9 & 0.64 & 4.5 & 2.5 \\
\hline
\end{tabular}

Table 2 - Non swollen ferrogels. FGA- $\boldsymbol{C L}=\mathbf{0 . 5} \%$ - Low field magnetooptical birefringence. $\Phi_{\text {synth }}$ volume fraction of nanoparticles in the ferrogel; $\tau_{\mathrm{o}}$ characteristic relaxation time; $\Phi_{\text {synth }}^{\text {rot }}$ and $\Phi_{\text {synth }}^{\text {block }}$ volume fractions of respectively rotating and blocked nanoparticles. For FFA dilute ferrofluids, we obtain $\tau_{o}^{F F}=5.4 \mu$ s (see Table SI.2 of SI).

The decay of intensity can be adjusted by a stretched exponential of relaxation time $\tau_{\mathrm{o}}$ which stretched exponent $\alpha$ is always close to 0.75 , smaller than that of the pure ferrofluid which is close to $\sim 1$. An advanced data treatment, presented in SI, enables to compare quantitatively the time distribution of rotating nanoparticles within ferrogels to the ones in the ferrofluid (see figure SI.4 of SI). It shows that in these unswollen ferrogels only short times are remaining. Nanoparticles of longer relaxation time seem to be blocked by the polymer within the ferrogels.

At low volume fractions $\Phi_{\text {synth }}$ of nanoparticles $\left(\Phi_{\text {synth }}=1 \%\right.$ and $3 \%, R_{\Pi}<0.1$ ), a large amount of nanoparticles is blocked by the presence of the polymeric chains. Let us note however that, at these $\Phi_{\text {synth }}$, the proportion of nanoparticles free to rotate $\Phi_{\text {synth }}^{\text {rot }} / \Phi_{\text {synth }}$ in the ferrogels at the end of synthesis is roughly equal to $\mathrm{R}$ the release ratio of nanoparticles during the swelling process (see table 2 and figure 4 ). When $\mathrm{R}_{\Pi}$ becomes large, this is no more true, namely at $\Phi_{\text {synth }}=7 \%\left(\mathrm{R}_{\Pi} \sim 0.5\right)$, $\Phi_{\text {synth }}^{\text {rot }} / \Phi_{\text {synth }}$ is found significantly larger than R.

\section{b) Rheological probing at swelling equilibrium}

We study here the rheological properties of ferrogels after swelling when the synthesis is at thermodynamic equilibrium. We perform here both static magneto-optical experiments in high magnetic fields and dynamic magneto-optical experiments in low magnetic fields. If both experiments can provide the amount of particles blocked in the ferrogels, one has to keep in mind that magnetic energy applied to a nanoparticle to produce its mechanical rotation are very different. In high fields experiments, it is of the order of its magnetic anisotropy energy, namely here $\sim 2 \mathrm{kT}_{\mathrm{amb}}{ }^{21}$ The blocked nanoparticles in high field experiments should thus be strongly coupled to the polymeric network, at least through an energy $8.10^{-21} \mathrm{~J}$.

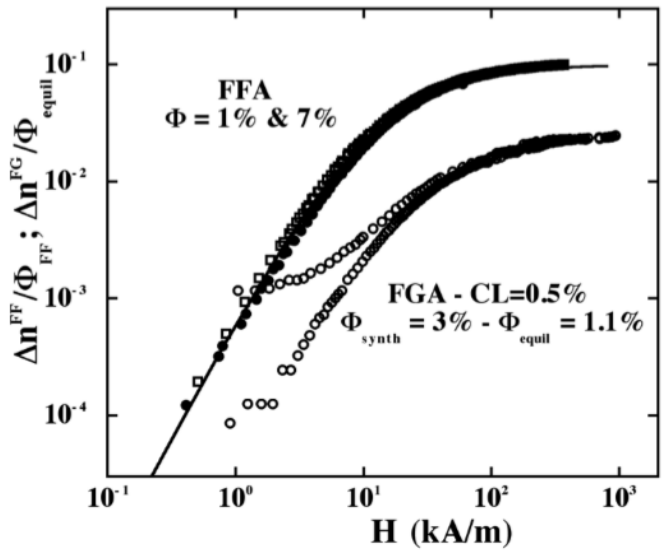

Figure 6 - Reduced birefringence $\Delta n^{F F} / \Phi^{F F}$ of ferrofluids FFA at $\Phi=1 \%$ (solid circles) and 7\% (open squares) - full line best fit by Langevin formalism (see part 2 of SI); $\Delta n^{F G} / \Phi_{\text {equil }}$ of ferrogel FGA at swelling equilibrium (at $\mathrm{CL}=0.5, \Phi_{\text {synth }}=3 \%, \Phi_{\text {nequil }}=1.1 \%$ ) as a function of applied field $H$.

Figure 6 plots the birefringence $\Delta n^{F G}$ as a function of magnetic field for a ferrogel at $\Phi_{\text {equil }}=1.1 \%\left(\Phi_{\text {synth }}=3 \%\right)$ and compares it to that of pure ferrofluid $\Delta n^{F F}$, normalized by the volume fraction inside the medium. Even though, both curves saturate in high fields, however they saturate at different values $\Delta \mathrm{n}_{\mathrm{S}} / \Phi$. In the ferrogel it is equal to 0.024 to be compared to the corresponding value 0.1 in ferrofluid FFA (see table SI.2 in SI). This difference comes from the fact that inside the ferrogel a significant amount of nanoparticles is unable to rotate mechanically within ferrogel under the action of a high magnetic field. In the ferrogel, $76 \%$ of the nanoparticles are tightly blocked by the polymeric network at $10^{3} \mathrm{kA} / \mathrm{m}$.

Moreover, a hysteresis is observed when the field is decreased back to zero. At the time scale of the experiment (about $15 \mathrm{mn}$ for the field decrease), an important proportion of nanoparticles keeps their mechanical orientation in zero field. This hysteresis disappears within a few hours denoting the existence of slow relaxations in the swollen system. The mechanical rotation of the nanoparticles are thus hindered by the polymers inside these ferrogels, even in fields as high as $10^{3} \mathrm{kA} / \mathrm{m}$.

\begin{tabular}{|c|c|c|c|c|c|c|c|c|}
\hline \multirow{2}{*}{$\begin{array}{c}\Phi_{\text {syn }} \\
\text { th } \\
(\%)\end{array}$} & \multirow{2}{*}{$\begin{array}{c}\Phi_{\text {equil }} \\
(\%)\end{array}$} & \multicolumn{2}{|c|}{$\Phi_{\text {equil }}^{\text {rot }} / \Phi_{\text {synth }}$} & \multicolumn{2}{|c|}{$\Phi_{\text {equil }}^{\text {rot }}(\%)$} & \multicolumn{2}{|c|}{$\Phi_{\text {equil }}^{\text {block }}(\%)$} & \multirow{2}{*}{$\tau_{\text {o }}(\mu \mathrm{s})$} \\
\cline { 5 - 9 } & HF & LF & HF & LF & HF & LF & LF \\
\hline 1 & 0.5 & 0.34 & 0.05 & 0.17 & 0.02 & 0.33 & 0.48 & 4.4 \\
\hline 3 & 1.1 & 0.24 & 0.10 & 0.26 & 0.11 & 0.84 & 0.99 & 3.5 \\
\hline 7 & 3.0 & 0.14 & 0.21 & 0.42 & 0.63 & 2.58 & 2.37 & 2.5 \\
\hline
\end{tabular}

Table 3 - Ferrogels at swelling equilibrium. FGA-CL=0.5\% Magneto-optical birefringence. $\Phi_{\text {synth }}$ initial volume fraction; $\Phi_{\text {equil }}$ volume fraction at swelling equilibrium; $\tau_{\mathrm{o}}$ characteristic relaxation time; $\Phi_{\text {equil }}^{\text {rot }}$ and $\Phi_{\text {equil }}^{\text {block }}$ volume fractions of respectively rotating and blocked nanoparticles. HF refers to High-Field experiments and LF to Low-Field experiments.

Table 3 reports the respective volume fractions of blocked particles and of particles free to rotate within the ferrogel for $\Phi_{\text {equil }}=0.5 \%, 1.1 \%$ and $3 \%$. After swelling, the volume fraction $\Phi_{\text {equil }}^{\text {rot }}$ of blocked nanoparticles in high field is close to $\Phi_{\text {equil. }}$ The volume fractions of rotating nanoparticles obtained in low fields in dynamic magneto-optical experiments are very 
This document is the Accepted Manuscript version of a Published Work that appeared in final form in Soft Matter, 2009, 5, 2614-2624, copyright (C) Royal Society of Chemistry after peer review and technical editing by the publisher. To access the final edited and published work see http://pubs.rsc.org/en/Content/ArticleLanding/2009/SM/b819189a

similar to the ones deduced from high fields measurements (table 3). It shows that the ratio of blocked nanoparticles in low field experiments corresponds to the particles coupled with the polymeric network. The characteristic times $\tau_{\mathrm{o}}$ of rotation remains short compared to the one of pure ferrofluid (see the distribution times in SI).

c) Comparison of ratios of nanoparticles blocked in ferrogel before and after swelling

If the nanoparticles, blocked before swelling, remain blocked at swelling equilibrium, it means that their number can be obtained with:

$$
\Phi_{\text {equil }}^{\text {block }}=\Phi_{\text {synth }}^{\text {block }} G_{\text {synth }} / G_{\text {equil }}
$$

The comparison of table 2 and 3 enables to check this assumption. We compare in table 4 the experimental values $\Phi_{\text {equil }}^{\text {block }}$ to the calculated ones (using equation 6). This is in very good accordance. The particles blocked just after synthesis remain blocked during swelling. It also means, because the volume fraction of polymer $\varphi_{\mathrm{AM}}$ is proportionnal to $1 / \mathrm{G}$ (see

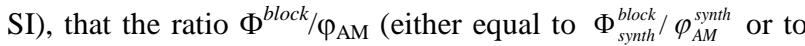
$\Phi_{\text {equil }}^{\text {block }} \varphi_{A M}^{\text {equil }}$ ) remains constant during the swelling process (see table 4).

\begin{tabular}{|c|c|c|c|c|c|}
\hline $\begin{array}{c}\Phi_{\text {synth }} \\
(\%)\end{array}$ & $\begin{array}{c}\Phi_{\text {synth }}^{\text {block }} \\
\text { meas. } \\
(\%)\end{array}$ & $\begin{array}{c}\Phi_{\text {equil }}^{\text {block }} \\
\text { meas. } \\
(\%)\end{array}$ & $\begin{array}{c}\Phi_{\text {equil }}^{\text {block }} \\
\text { calc. } \\
(\%)\end{array}$ & $\begin{array}{c}\Phi_{\text {sycth }}^{\text {block }} / \varphi_{A M}^{\text {synth }} \\
\text { meas. }\end{array}$ & $\begin{array}{c}\Phi_{\text {equil }}^{\text {block }} \\
\text { meas. }\end{array}$ \\
\hline 1 & 0.85 & 0.48 & 0.48 & 0.30 & 0.31 \\
\hline 3 & 2 & 0.99 & 1.08 & 0.72 & 0.66 \\
\hline 7 & 2.5 & 2.37 & 2.17 & 0.90 & 1.00 \\
\hline
\end{tabular}

Table 4 - FGA - $\boldsymbol{C L}=\mathbf{0 . 5} \%$ - Volume fraction of blocked nanoparticles in ferrogels at swelling equilibrium ( $\Phi_{\text {equil }}^{\text {block }}$ ) and in synthesis conditions $\left(\Phi_{\text {synth }}^{\text {block }}\right.$. Comparison between measurements of tables 2 and 3, and calculation with equation $6 ; \Phi_{\text {synth }}^{\text {block }} / \varphi_{A M}^{\text {synth }}$ : volume ratio of nanoparticles with respect to polymer in the synthesis conditions; $\Phi_{\text {equil }}^{\text {block }} / \varphi_{A M}^{\text {equil }}$ : volume ratio of nanoparticles with respect to polymer at swelling equilibrium.

d) SANS experiments and structure of ferrogels

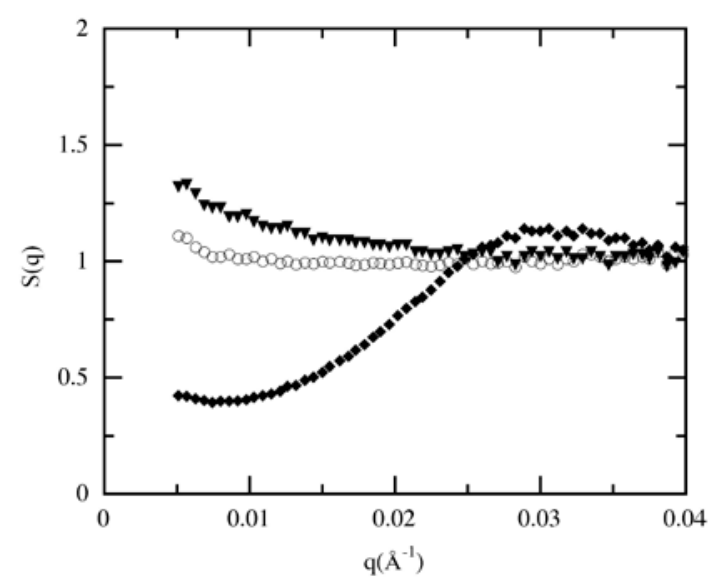

Figure 7 -Ferrogels at swelling equilibrium FGA - CL $=0.5 \%$ Structure factor of the nanoparticles $\boldsymbol{S}(\boldsymbol{q})$ versus q at $\Phi_{\text {equil }}=0.5 \%$ (solid triangles), 1.1\% (open circles) and 3\% (solid diamonds).

The structure factor $S(q, \Phi)$ of swollen ferrogels at various volume fractions are presented in figure 7 . They are obtained by dividing the scattering of ferrogels by the form factor of the ferrogels measured on pure ferrofluids. As almost all the particles remaining in the ferrogels are coupled to the polymeric network, the structure is not at all similar to that of a pure ferrofluid solution. It is the structure of a matched polymeric network decorated with nanoparticles. For $\Phi_{\text {equil }}=$ $0.5 \%$ and $1 \%$, the structure factor is roughly equal to 1 , except at low q where there is a slight upturn. The nanoparticles behave like a dilute system where nanoparticles are randomly organized with a low effective attraction.

At $\Phi_{\text {equil }}=3 \%$, which corresponds to a sample synthesized in concentrated regime $\left(\Phi_{\text {synth }}=7 \%\right)$, there is still the slight upturn at low q and a correlation peak at $0.032 \AA^{-1}(\sim 200 \AA$ in real space). This peak corresponds to the most probable interparticle distance inside the polymeric network. Let us assume that the polymeric network is homogeneous with a mesh size $\xi$. In the framework of this hypothesis, the initial mesh size before swelling would be here $170 \AA$ (see table 1 of SI) and would remain of the order of $170 \AA$ after swelling (see Fig 3). $\xi$ is of the same order of magnitude as the most probable interparticle distances. It is thus a rather homogeneous polymeric network that the nanoparticles are 'decorating'.

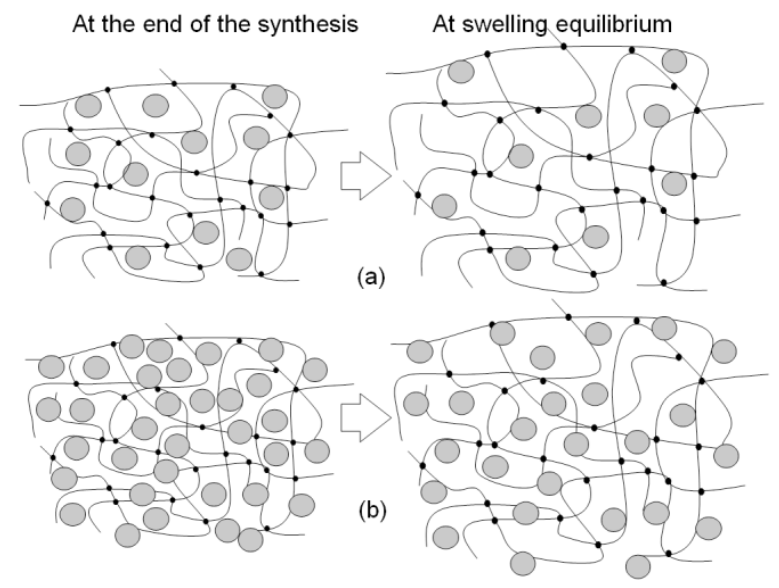

Figure 8 - Route $1: R_{S}<1$ - FFA CL $=0.5 \%$ - Pictures of gels at the end of the synthesis (left) and at swelling equilibrium (right), (a) low $\Phi_{\text {synth }}(1 \%$ or $3 \%)$; (b) high $\Phi_{\text {synth }}(7 \%)$.

Moreover the correlation peak corresponds also to the mean distance between nanoparticles in a pure ferrofluid solution at $3 \%$ in repulsive regime (see Fig SI.2). The particles fill thus all the available space in the swollen ferrogel and each mesh contains a nanoparticle. The covering of the polymeric network by nanoparticles is thus close to saturation. Note that if in this structure $80 \%$ of the nanoparticles are blocked in rotation, $20 \%$ still rotates as if in a liquid environment, without polymer hindrance, with a size of the order of the mesh of the network.

The structures of the ferrogels made with CL $=0.5 \%$ with FFA (at the end of the synthesis and at swelling equilibrium) are pictured in figure 8 for the whole range of $\Phi_{\text {synth }}$ ).

\section{Ferrogels at $R_{s} \sim 1$}

We study here the structure and properties of ferrogels after swelling when $\mathrm{R}_{\mathrm{S}} \sim 1$. We choose thus to work with FGB samples at CL $=2 \%$ (see table 1 ) and we vary $R_{\Pi}$ in a large range. As seen in Fig 3, there is no swelling at all for such samples: $G_{\text {equil }}$ remains very close to $G_{\text {synth }}$. However, there are two different releasing behaviors depending on $\Phi_{\text {synth. }}$ At low $\Phi_{\text {synth }}\left(1 \%\right.$ and 3\%), there is no release. Oppositely above $\Phi_{\text {synth }}$ $=3 \%$, there is a very large release, as $\Phi_{\text {equil }}$ decreases to a value of the order of $1 \%$. The following measurements enlighten these two very different behaviors 
This document is the Accepted Manuscript version of a Published Work that appeared in final form in Soft Matter, 2009, 5,

2614-2624, copyright (C) Royal Society of Chemistry after peer review and technical editing by the publisher. To access the final edited and published work see http:// pubs.rsc.org/en/Content/ArticleLanding/2009/SM/b819189a

a) Rheological probing at swelling equilibrium

We have deduced from low-field dynamical birefringence the volume fractions $\Phi_{\text {equil }}^{\text {rot }}$ of rotating nanoparticles and $\Phi_{\text {equil }}^{\text {block }}$ of blocked nanoparticles in the swollen ferrogels (see table 5). A large proportion of the nanoparticles is blocked whatever $\Phi_{\text {synth. }}$. Table 5 gives also the ratio $\Phi_{\text {equil }}^{\text {block }} / \varphi_{A M}^{\text {equil }}$ for each $\Phi_{\text {synth. }}$.

\begin{tabular}{|c|c|c|c|c|c|c|}
\hline $\begin{array}{c}\Phi_{\text {synth }} \\
(\%)\end{array}$ & $\begin{array}{c}\Phi_{\text {equil }} \\
(\%)\end{array}$ & $\begin{array}{c}\tau_{\mathrm{o}} \\
(\mu \mathrm{s})\end{array}$ & $\frac{\Phi_{\text {equil }}^{\text {rot }}}{\Phi_{\text {equil }}}$ & $\begin{array}{c}\Phi_{\text {equil }}^{\text {rot }} \\
(\%)\end{array}$ & $\begin{array}{c}\Phi_{\text {equil }}^{\text {block }} \\
(\%)\end{array}$ & $\begin{array}{c}\frac{\Phi_{\text {equil }}^{\text {block }}}{\varphi_{\text {AM }}^{\text {equil }}} \\
\text { meas. }\end{array}$ \\
\hline 1 & 0.97 & 4.4 & 0.05 & 0.05 & 0.92 & 0.33 \\
\hline 3 & 2.95 & 2.24 & 0.26 & 0.76 & 2.19 & 0.79 \\
\hline 5 & 0.75 & 3.6 & 0.08 & 0.06 & 0.69 & 0.25 \\
\hline 7 & 0.82 & 3.43 & 0.26 & 0.22 & 0.60 & 0.22 \\
\hline
\end{tabular}

Table 5 - Ferrogels at swelling equilibrium. FGB-CL=2\% - low field magneto-optical birefringence.- Same notations as in table 3

There is a majority of blocked nanoparticles at any $\Phi_{\text {synth }}$. However in the samples at $\Phi_{\text {synth }}=3 \%$ and $7 \%$, about $~ 25 \%$ of the amount of nanoparticles entrapped in the swollen system are still rotating. This is clearly different from the case where $\mathrm{R}_{\mathrm{s}}<1$ where most of the particles free to rotate at the end of the synthesis are released during the swelling process since almost all of the particles remaining in ferrogels are blocked at swelling equilibrium. This is indeed related here to the tight mesh size of the network when $\mathrm{R}_{\mathrm{s}} \sim 1$. Moreover the characteristic time $\tau_{\mathrm{o}}$ of the rotating nanoparticles is here still of the same order of magnitude as $\tau_{o}^{F F}$ (the relaxation time in the pure ferrofluid - see Table SI.2) and the stretched exponent has a constant value of $\sim 0.9$, also close to the one of the pure ferrofluid. It shows that the movement of the rotating nanoparticles is not strongly hindered by the polymeric network nor related to the existence of large nanoparticle agglomerates. These rotating nanoparticles should be located in a liquid environment within the sample. It enables us to conclude that the network is not homogeneous. Indeed, since $\mathrm{R}_{\mathrm{s}}$ $\sim 1$ and since the ferrogel does not swell, an homogeneous network is not compatible with particles in a liquid environment.

At $\Phi_{\text {synth }}>3 \%$, the very strong release of nanoparticles from ferrogels that do not swell implies the existence of large inhomogeneities within the synthesized samples. The volume fraction of blocked nanoparticles being quite constant in these swollen samples, it also suggests a saturation of the fraction of the polymeric network accessible to the nanoparticles.

\section{b) SANS experiments and structure of ferrogels}

The SANS structure factors of the four ferrogels are presented in Figure 9. At low $\Phi_{\text {synth }}(1 \%) \mathrm{S}(\mathrm{q})$ is rather flat, while at larger $\Phi_{\text {synth }}$, S(q) presents a slight upturn at low q together with a maximum at a wave-vector $\mathrm{q}_{\max }=2 \pi / \mathrm{l}_{\mathrm{mp}}$ associated with the most probable interparticle distance $\mathrm{l}_{\mathrm{mp}}$.

For sake of reasoning, let us first assume that the polymer network is here homogeneous. It would lead to a mesh size $\xi_{\text {hom }}$ $\sim 110 \AA$ (see table SI.1) very close to the size of the nanoparticles. A network decorated with one nanoparticle per mesh (as in part 3) would present a maximum of $\mathrm{S}(\mathrm{q}$ ) at $2 \pi / \xi_{\text {hom }} \sim 0.057 \AA^{-1}$ (see inset of figure 9 - dashed line) much larger than the experimental $\mathrm{q}_{\max }=2 \pi / \mathrm{l}_{\mathrm{mp}}$ (open circles). This is in any case not possible because it would imply much larger volume fractions of nanoparticles than the present ones.

Let us now assume that the spatial distribution of nanoparticles in the ferrogel is homogeneous with a mean interparticle distance $l_{\text {mean }}(\Phi)=d_{o} \sqrt[3]{\frac{\pi}{6 \Phi}}$. The maximum of $\mathrm{S}(\mathrm{q})$ will then be located at $\mathrm{q}_{\text {mean }}(\Phi)=2 \pi / \mathrm{l}_{\text {mean }}=\frac{2 \pi}{d_{o}} \sqrt[3]{\frac{6 \Phi}{\pi}}$, which is plotted as a full line in inset of Fig. 9. Surprisingly, in these ferrogels, $\mathrm{q}_{\max }$ is experimentally very close to $2 \pi / \mathrm{l}_{\text {mean }}\left(\Phi_{\text {synth }}\right)$ (and for $\Phi_{\text {synth }}>3 \%$ sensibly larger than what would be expected for $\left.2 \pi / l_{\text {mean }}\left(\Phi_{\text {equil }}\right)\right)$. It allows to conclude that for:

- the spatial distribution of nanoparticles is clearly heterogeneous in the ferrogel.

- $l_{\text {mean }}\left(\Phi_{\text {synth }}\right)$ is close to $l_{\text {mp }}$, even if slightly larger. The swollen ferrogel has a kind of "memory" of the initial large volume fraction of nanoparticles in the synthesis bath.

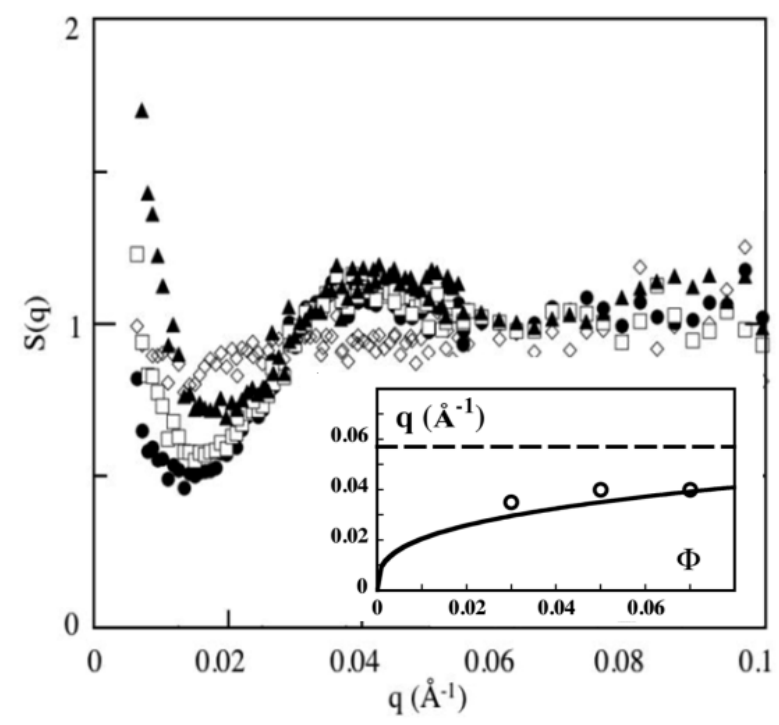

Figure 9 - Ferrogels at swelling equilibrium. FGB CL $=2 \%$ Structure factor $\mathrm{S}(\mathrm{q})$ versus $\mathrm{q}$ at $\Phi_{\text {synth }}=1 \%(\Gamma), \Phi_{\text {synth }}=3 \%(\lambda)$; $\Phi_{\text {synth }}=5 \%$ (open squares); $\Phi_{\text {synth }}=7 \%(\sigma)$. Inset $-\mathrm{q}$ versus $\Phi$ (Dashed line : $\mathrm{q}_{\max }=2 \pi / \xi_{\text {hom }}$; Full line : $\mathrm{q}_{\text {mean }}$ versus $\Phi$; Open circles : experimental $\mathrm{q}_{\max .}=2 \pi / \mathrm{l}_{\mathrm{mp}}$ from fig.9a versus $\left.\Phi_{\text {synth }}\right)$

The increase of the structure factor at low q observed for $\Phi_{\text {synth }} \geq 3 \%$ also shows that the nanoparticles are here organized in a very heterogeneous way within the ferrogels. There are areas of the ferrogel in which there is a high density of nanoparticles and areas empty of nanoparticles. In the areas of the ferrogel rich in nanoparticles, two situations can be considered: (i) the nanoparticles are locally concentrated or (ii) the nanoparticles form large aggregates. This second situation is not possible because we do not observe a correlation peak corresponding to the contact of two particles at $2 \pi / \mathrm{d}_{0} \sim 0.08 \AA^{-1}$ which should be obtained in case of the presence of dense aggregates. ${ }^{22}$ There are thus spatial regions of the ferrogels where nanoparticles have been concentrated.

Since a large amount of the nanoparticles is coupled with the polymeric network inside the swollen ferrogel, the heterogeneous spatial distribution of nanoparticles shows that the polymeric network is also heterogeneous with regions of high polymer density coexisting with regions of low polymer density. The mass conservation of the polymer implies then that there are some regions of the ferrogel where the mesh size 
of the network is inferior to the diameter of the nanoparticles. These parts are thus not accessible to the nanoparticles. The structure of the samples is thus composed of: (i) some regions with a high density of polymer and a small mesh size without nanoparticles, (ii) some regions where the nanoparticles are linked to the polymeric network and (iii) some pockets where there are no polymers and eventually some free nanoparticles in a fluid environment.
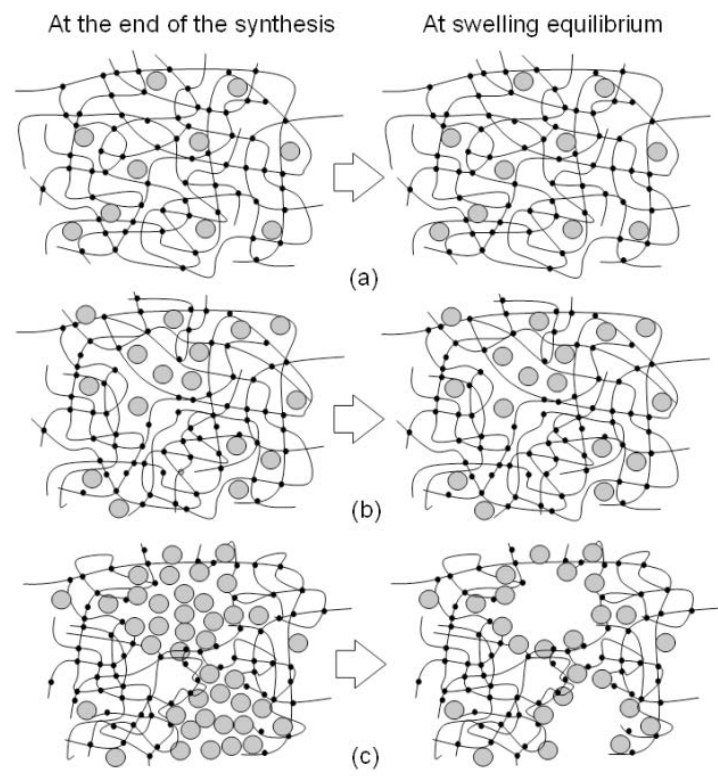

Figure 10 - Route $2: R_{S} \sim 1$ - FFB $C L=2 \%$ - Pictures of gels at the end of the synthesis (left) and at swelling equilibrium (right), (a) low $\Phi_{\text {synth }}(1 \%)$; (b) intermediate $\Phi_{\text {synth }}(3 \%)$; (c) high $\Phi_{\text {synth }}(5 \%$ or $7 \%$ ).

At $\Phi_{\text {synth }} \geq 3 \%$, the presence of liquid pockets containing nanoparticles may explain why we observe a correlation peak. The situation of the nanoparticles in the pockets is close to the one observed with pure ferrofluid solutions with a concentration of the order of $\sim 7-8 \%$ (at $\Phi_{\text {synth }}>3 \%$ ). It leads to a correlation peak at the same wavevector (see the scattering spectra of the pure FFB ferrofluid at $\Phi=7 \%$ in figure SI.2), but with an interparticle distance less defined. However in these systems, the total amount of rotating nanoparticles is at most $25 \%$ (of $\Phi_{\text {equil), }}$, the majority of the nanoparticles is blocked in rotation and thus linked to the polymeric network. Taking into account the observed intensity of the correlation peak, these blocked nanoparticles must also contribute to the peak and they thus should decorate the polymeric network with the same most probable distance. In this context, we can easily imagine that, during the swelling process, the pockets of the already very heterogeneous networks can percolate favoring the release of nanoparticles. This process then leaves behind a decoration of (blocked) nanoparticles on the surface of the empty pockets with the same interparticle distance as the ferrofluid previously contained in these pockets. This explains in the swollen ferrogels (prepared with $\Phi_{\text {synth }}=5 \%$ and $\Phi_{\text {synth }}=7 \%$ ) : (i) their large release ratio (see Fig. 4), (ii) their low value of $\Phi_{\text {equil }}$ because the polymeric network outside the pockets is very tight, (iii) their large proportion of blocked nanoparticles and (iv) the potential remaining of some isolated fluid pockets.

If we assume that at $\mathrm{R}_{\mathrm{S}} \sim 1$, the particles blocked after the synthesis remain blocked after the swelling process as it is observed at $\mathrm{R}_{\mathrm{S}}<1$, it would also explain the rather low proportion of blocked nanoparticles at the end of the synthesis. The blocked nanoparticles are mainly decorating the pockets and are not introduced in the regions with a tight polymeric network. Indeed at $\Phi_{\text {synth }}=3 \%$, decorated pockets full of nanoparticles in a liquid environment also exist but remain isolated. The nanoparticles cannot be released because they cannot cross the regions where the polymeric mesh size is lower than their diameter. The pocket decoration confirms the existence of a specific interaction between the nanoparticles.

The structures of FGB ferrogels at CL $=2 \%$ (at the end of the synthesis and after swelling) are pictured in figure 10 for the whole investigated range of $\Phi_{\text {synth}}$.

\section{5 - Discussion}

The different local structures of ferrogels obtained in parts 3 and 4 shows that the homogeneity of ferrogels is controlled by the synthesis conditions and is tuned by both $\mathrm{R}_{\mathrm{S}}$ and $\mathrm{R}_{\Pi}$. Indeed as the polymeric network is cross-linked by covalent bonds its homogeneity cannot change during swelling.

$R_{S}$ is the main parameter that influences the homogeneity of the ferrogel in the synthesis conditions. If $\mathrm{R}_{\mathrm{S}}<1$, the nanoparticles can easily enter in the meshes of the hydrogel without any distortion. Oppositely, for $R_{S}>1$ an entrapped nanoparticle will necessary forces the mesh size in its immediate surrounding to be larger than the average mesh. The presence of the nanoparticles will thus bring inhomogeneities within the whole ferrogel. The difference of release ratios between FGA and FGB samples, at CL $=2 \%$ and low $\Phi_{\text {synth }}$, illustrates this point. Samples made with FFA release nanoparticles though samples made with FFB do not. For such a high cross-linking ratio, the polymeric network is heterogeneous with zones of pure polymer network and pockets of ferrofluid. The zones of pure polymeric network are so dense that the diffusion of free nanoparticles through them is impossible. Nanoparticles can only be released during swelling if they can follow an open percolating path. This happens in FGA samples $\left(R_{S}>1\right)$ not in FGB $\left(R_{S} \sim 1\right)$ where the heterogeneities are less pronounced. It allows to sort out two families of ferrogel structures.

If $\mathrm{R}_{\mathrm{S}}<1$, the polymeric network is weakly modified by the nanoparticles and the ferrogel is homogeneous. This is the situation observed whatever $\Phi_{\text {synth }}$, as long as $R_{\Pi}<0.5$. For $R_{\Pi}$ $\sim 0.5$, the polymeric network becomes slightly heterogeneous. There are some nanoparticles able to rotate within the ferrogel. This is the situation for FGA at $\Phi_{\text {synth }}=7 \%$ and a cross-linking ratio $\mathrm{CL}=0.5 \%$.

If $R_{S} \geq 1$, the system is heterogeneous. At low $R_{\Pi}$, there may be some nanoparticles able to rotate within the ferrogel in ferrofluid pockets. This is the situation for FGB at $\Phi_{\text {synth }}=3 \%$ and $\mathrm{CL}=2 \%$, with $\mathrm{R}_{\Pi} \sim 0.2$. For $\mathrm{R}_{\Pi}>0.2$ the polymeric network is very heterogeneous at the synthesis. The pockets of ferrofluid percolate to form continuous zones, free of polymer, within the ferrogel, that can empty during the swelling process. This explains the large release ratio observed with FGB ferrogels at CL $=2 \%$ for $\Phi_{\text {synth }}=5 \%$ and $\Phi_{\text {synth }}=7 \%$. This is also the reason why FGA ferrogels at CL $=2 \%$ and $\Phi_{\text {synth }} \geq 5 \%$ break down during swelling.

Apart from the conditions that play on the homogeneity of the ferrogel during the synthesis, a very important feature of the ferrogels is evidenced here whatever their homogeneity. It is an attractive interaction between the nanoparticles and the polymer chains that induces a strong adsorption of the nanoparticles on the polymer network. The existence of an attractive interaction is clearly proved by the reduction of the swelling ratio of the ferrogel compared to the swelling ratio of 
This document is the Accepted Manuscript version of a Published Work that appeared in final form in Soft Matter, 2009, 5,

2614-2624, copyright (C) Royal Society of Chemistry after peer review and technical editing by the publisher. To access the final edited and published work see http://pubs.rsc.org/en/Content/ArticleLanding/2009/SM/b819189a

the hydrogel at the same cross-linking ratio CL. Without interaction between the nanoparticles and the polymeric chains, the addition of nanoparticles bearing a negative charge in the hydrogel would have increased the chemical potential of water inside the gel and thus the swelling of the hydrogel (like in a dialysis bag). As one observes exactly the opposite behavior even at low $\Phi_{\text {synth }}$, it points out an attractive part in the sum of interaction between nanoparticles and polymer chains. When $\Phi_{\text {synth }}$ is increased in the network, the effect increases and the swelling ratio $G_{\text {equil }}$ of the ferrogel decreases (see Figure 4).

These attractive interactions existing between iron oxide nanoparticles and the PAM chains in the ferrogels are ascribed to hydrogen bonds between the carbonyl groups of the polymer (each monomer bearing a primary amide function) and hydroxyl functions at the iron oxide surface, either $\mathrm{Fe}-\mathrm{OH}$ directly at the solid surface or hydroxyl end-groups from the citrate ligands coating the nanoparticles. The hypothesis of $\mathrm{H}-$ bonds formation between PAM and many oxides was proposed long ago. ${ }^{23}$ It was evidenced by techniques like the shift of infra-red absorption bands of the polymer ${ }^{24}$ and the shift of the iso-electric point of the oxide surface measured by potentiometric titration and zetametry. ${ }^{25}$ Such H-bonds were also invoked in the kinetics of adsorption of PAM onto the haematite $\left(\alpha-\mathrm{Fe}_{2} \mathrm{O}_{3}\right)$ surface, which has a chemical reactivity close to maghemite $\left(\gamma-\mathrm{Fe}_{2} \mathrm{O}_{3}\right){ }^{26}$ The completely different mechanism of complexation by direct chelation of $\mathrm{Fe}^{\mathrm{III}}$ ions by polar groups of the polymer is very unlikely in our case of ferrogels, because the iron oxide surface is already covered by a dense layer of citrates, which offer many hydroxyl moities to establish H-bonds with the polymer.

A second experimental evidence of this interaction is given by the observation of blocked nanoparticles just after synthesis, remaining blocked all along the swelling process, even if the mesh is larger than the nanoparticles $\left(R_{S}<1\right)$. As a release is geometrically possible, the remaining nanoparticles are thus all adsorbed to the polymeric network and decorate it. Depending on the synthesis conditions they are then decorating either a homogeneous polymer network $\left(\mathrm{R}_{\mathrm{S}}<1\right)$ or the pockets (empty or full of ferrofluid) of an heterogeneous network $\left(\mathrm{R}_{\mathrm{S}} \sim 1\right)$. The birefringence experiment in large fields gives an indication about the binding energy of the nanoparticles to the polymeric network : it is larger than $2 \mathrm{kT}_{\mathrm{amb}}$, a hydrogen bond being of the order of $4 \mathrm{kT}_{\mathrm{amb}}$. Figure 11 quantifies the adsorption of the nanoparticles on the polymer chains through the $\Phi_{\text {synth }}{ }^{-}$ dependence of the ratio $\Phi^{\text {block}} / \varphi_{\mathrm{AM}}$, volume fraction of blocked nanoparticles over polymeric volume fraction. It collects for homogeneous networks the previous observations of Tables 4 and 5:

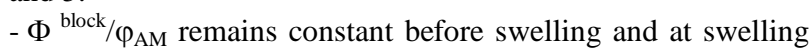
equilibrium, as adsorbed nanoparticles cannot diffuse outside the gel,

- the cross-linking ratio has no influence on $\Phi^{\text {block} /} / \varphi_{\mathrm{AM}}$, as the adsorption process is indeed related to an interaction between the polymeric chains and the nanoparticles,

- $\Phi^{\mathrm{block}} / \varphi_{\mathrm{AM}}$ only depends on $\Phi_{\text {synth }}$ and saturates at large $\Phi_{\text {synth }}$, this plateau being consistent with SANS experiments on FGA at CL $=0.5 \%$ and $\Phi_{\text {synth }}=7 \%$ that shows that the covering of the polymeric network by nanoparticles is close to saturation.

In the two heterogeneous swollen samples FGB at $\mathrm{CL}=2 \%$ and large $\Phi_{\text {synth }}$, the values of $\Phi_{\text {equil }}^{\text {block }} \varphi_{A M}^{\text {equil }}$ can no longer be located on the absorption curve of figure 11 because a large part of the heterogeneous network is not accessible to the nanoparticles and does not participate to the adsorption process (see diamonds at large $\Phi_{\text {synth }}$ in Fig. 11).

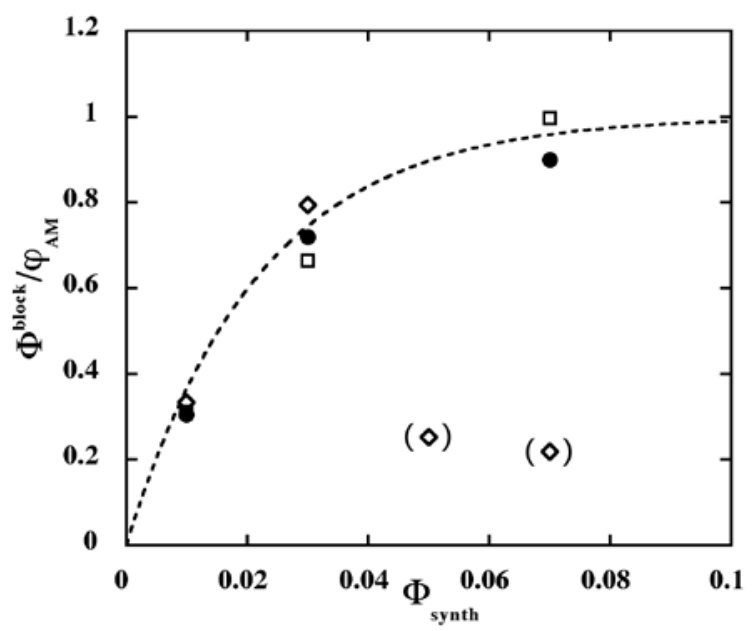

Figure 11 - Nanoparticle adsorption - Volume ratio of nanoparticles with respect to polymer in the synthesis conditions $\Phi_{\text {synth }}^{\text {block }} \varphi_{A M}^{\text {synth (full }}$ circles: FGA - CL $=0.5 \%$ ) and at swelling equilibrium $\Phi_{\text {equil }}^{\text {block }} / \varphi_{A M}^{\text {equil }}$ (open squares : FGA - CL $=0.5 \%$; open diamonds : FGB $-\mathrm{CL}=2 \%$ ) versus initial volume fraction of nanoparticles $\Phi_{\text {synth }}$.

Nanoparticle adsorption also limits the nanoparticle release during swelling, however it is not always the only cause for a low release. Because of local heterogeneities in the polymeric network, pockets of ferrofluid, with nanoparticles able to rotate, can be entrapped in the ferrogel even after swelling (see for example FGB at $\mathrm{CL}=2 \%$ and $\Phi_{\text {synth }}=3 \%$ ).

This strong adsorption of the nanoparticles on the polymeric chains can explain the large mechanical reinforcement obtained in ${ }^{9}$ with FGA ferrogels at CL=1\%. In such ferrogels, the synthesis conditions were chosen to get a ferrogel decorated by the nanoparticles with an homogeneous structure $\left(\mathrm{R}_{\mathrm{S}}<1\right.$ and $\left.\mathrm{R}_{\Pi}<1\right)$. It leads to a relative increase of the Young modulus $\mathrm{E}^{\mathrm{FG}}-\mathrm{E}^{\mathrm{HG}} / \mathrm{E}^{\mathrm{HG}}$ varying exponentially with $\Phi_{\text {equil }}$ $/ \varphi_{\mathrm{AM}}$ (see inset of Figure 12 ) reaching for $\Phi_{\text {synth }}=7 \%\left(\Phi_{\text {equil }}\right.$ $=3 \%$ ) a value 10 times larger than that of the corresponding hydrogel. It means that the number of cross-links mediated by the nanoparticles is then 10 times larger than the BAM crosslinks.

Let us note as a last point that in the ferrogel (as it is true in the hydrogel - see SI) the Young modulus at swelling equilibrium is directly proportional to $\Pi_{\text {mix }}^{F G}$ the osmotic pressure of mixing of all the components of the system. By substracting $\Pi_{\text {mix }}^{H G}$ the mixing osmotic pressure of the pure hydrogel (see SI), it is then possible to deduce the osmotic pressure $\Pi_{\text {mix }}^{\text {nanop }}$ associated to the mixing of the nanoparticles in the system "polymer + solvent". These determinations for FGA at $\mathrm{CL}=1 \%$ are plotted in figure 12 as a function of $\Phi_{\text {equil }}$. Adjusted by a Carnahan-Starling formalism (see SI), a much larger repulsion range $(\delta=12 \mathrm{~nm})$ is found with respect to that of $\Pi^{F F}$ of the initial ferrofluid FFA $(\delta=4.5 \mathrm{~nm})$. In these homogeneous ferrogels, the nanoparticles are distributed as in a dispersion of very strongly repulsive Hard-Spheres, this effective repulsion being mediated by the polymer chains on which the nanoparticles are adsorbed. This let suspect only weak effects from an applied magnetic field, as in standard strongly repulsive ferrofluids ${ }^{27}$, a softening of the structure 
This document is the Accepted Manuscript version of a Published Work that appeared in final form in Soft Matter, 2009, 5, 2614-2624, copyright (C) Royal Society of Chemistry after peer review and technical editing by the publisher. To access the final edited and published work see http://pubs.rsc.org/en/Content/ArticleLanding/2009/SM/b819189a

being necessary to make them observable. As in ferrofluids, such effects could be induced if the ionic strength is increased either in the synthesis conditions or in the swelling bath.

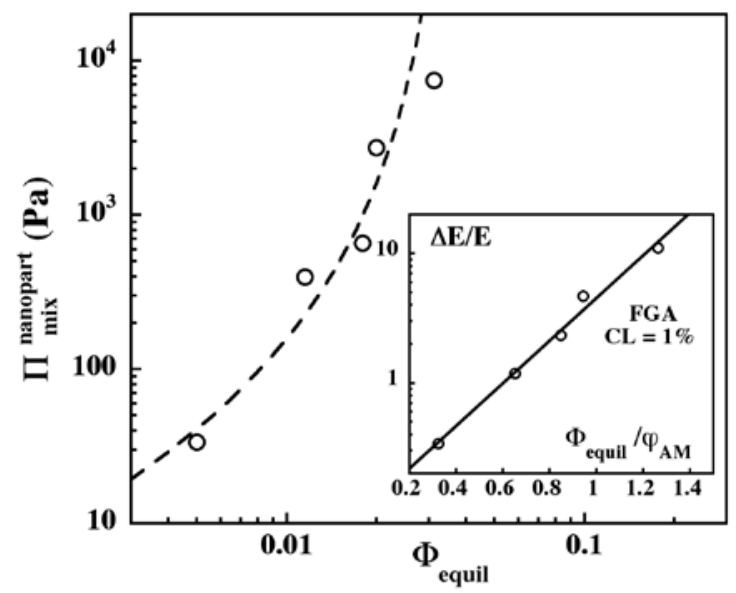

Figure 12 - Homogeneous ferrogels FGA at swelling equilibrium $\boldsymbol{C L}=\mathbf{1 \%}$ - Nanoparticle contribution $\Pi_{\text {mix }}^{\text {nanopart }}$ to mixing pressure of ferrogels versus $\Phi_{\text {equil }}$ (open circles - dashed line: its Carnahan-Starling adjustment). Inset: Reduced variations $\left(\mathrm{E}^{\mathrm{FG}}-\mathrm{E}^{\mathrm{HG}}\right) / \mathrm{E}^{\mathrm{HG}}$ of the elastic modulus (from ${ }^{9}$ ) in these swollen ferrogels as a function of $\Phi_{\text {equil }} / \varphi_{\mathrm{AM}}$ in a log-log representation. The full line is an exponential fit.

\section{Summary and perspectives}

Magnetic nanocomposite materials of low Young modulus are here synthesized by dispersing maghemite nanoparticles in a PAM polymeric network. We show how the local structure of a ferrogel can be tuned from a very homogeneous structure to a very heterogeneous one by the synthesis conditions. These local structures are elucidated here thanks to the combined use of physico-chemical determinations, of magneto-optical dynamic birefringence and of static Small Angle Neutron Scattering. These measurements reveal an adsorption of the nanoparticles on the polymeric chains, which allows keeping some nanoparticles inside the swollen gels. This adsorption limits the swelling process and decorates the polymeric network with nanoparticles, which explains the reinforcement observed in ${ }^{9}$ with these ferrogels at $\mathrm{CL}=1 \%$ because entrapped nanoparticles behave as supplementary cross-linkers in the network. If the ratio $\mathrm{R}_{\mathrm{S}}$ (defined as the nanoparticle effective volume over the polymeric mesh one in the homogeneous hydrogel) is too large the system becomes very heterogeneous and decorated pockets are observed. If $\mathrm{R}_{\mathrm{S}}<1$, a homogeneous network decorated at the spatial scale of the polymeric mesh is observed for $R_{\Pi} \leq 1$. When the ratio of volume fraction of nanoparticles to volume fraction of polymer $\Phi_{\text {equil }} / \varphi_{\text {AM }}$ reaches 1 , the polymeric array is saturated. If $R_{S} \geq 1$, it is no longer possible to form homogeneous ferrogels during the synthesis. The ferrogels are formed by connected networks of polymers with a tight mesh size without nanoparticles coexisting with pockets free of polymers decorated by nanoparticles. It becomes then difficult to keep the properties of the ferrogels under control.

We have thus shown how it is possible to monitor the mechanical properties of the ferrogels. As the key point of the monitoring of the properties is the interaction between the different partners of this complex system, we will investigate in a further work how the salinity of the medium modifies this interaction and how it can change the properties of these swollen ferrogels.

\section{Acknowledgements}

We thank Delphine Talbot for the synthesis of the ferrofluids. This work was supported by CONACYT Mexico.

\section{References}

[1] Z. Varga, G. Filipcsei, M. Zrinyi, Polymer 47 (2006) 227

[2] S. Abramchuk, E. Kramarenko, G. Stepanov, L.V. Nikitin, G. Filipcsei, A.R. Khokhlov, M. Zrinyi, Polym. Adv. Technol. 18 (2007) 883

[3] J. Oberdisse, P. Hine, W. Pyckhout-Hintzen, Soft Matter 3 (2007) 476

[4] J. Oberdisse, A. El Harrak, G. Carrot, J. Jestin, F. Boué, Polymer 46 (2005) 6695

[5] J. Jestin, F. Cousin, I. Dubois, C. Ménager, J. Oberdisse, R. Schweins, F. Boué, Adv. Mat. 20 (2008) 2533-2540

[6] J. Shigeura et al, US Patent 5338426 (1994)

[7] A.V. Texeira, I. Morfin, F. Ehrburger-Dolle, C. Rochas, E. Geissler, P. Licinio, P. Panine, Phys. Rev. E 67 (2003) 021504

[8] R. Shenhar, T.B. Norsten, V.M. Rotello, Adv. Mater. 16 (2005) 657-669

[9] J.A. Galicia, O. Sandre, F. Cousin, D. Guemghar, C.

Ménager, V. Cabuil, J. Phys.: Condens. Matter 15 (2003)

S1379

[10] C. Rivière, C. Wilhelm, F. Cousin, V. Dupuis, F. Gazeau,

R. Perzynski, Eur.Phys.J. E 22 (2007) 1

[11] S. Lecommandoux, O. Sandre, F. Checot, R. Rodriguez-

Hernandez, R . Perzynski, Advanced Mat. 17 (2005) 712

[12] G. Mériguet, E. Dubois, V. Dupuis, R. Perzynski, J. Phys.: Condens. Matter 18 (2006) 10119

[13] C. Wilhelm, F. Gazeau, J. Roger, J.N. Pons, M.F. Salis, R. Perzynski, J.-C. Bacri, Phys. Rev. E 65 (2002) 031404

[14] E. Hasmonay, A. Bee, J.-C. Bacri, R. Perzynski, J Phys

Chem B 103 (1999) 6421-6428

[15] E. Dubois, V. Cabuil, F. Boué, R. Perzynski, J. Chem. Phys. 111 (1999) 7147

[16] F. Cousin, E. Dubois, V. Cabuil, Phys. Rev. E 68 (2003) 021405

[17] L. Benguigui, F. Boué, Eur. Phys. J. B 11 (1999) 439

[18] D. Asnaghi, M. Giglio, A. Bossi, P.G. Righetti

Macromolecules 30 (1997) 6194

[19] F. Gazeau, F. Boué, E. Dubois, R. Perzynski, J. Phys.:

Condens. Matter 15 (2003) S1305

[20] E. Hasmonay, E. Dubois, J.-C. Bacri, R. Perzynski, Yu. L.

Raikher, V.I. Stepanov, Eur. Phys. J. B 5 (1998) 859

[21] F. Gazeau, J.-C. Bacri, F. Gendron, R. Perzynski, Yu.

Raikher, V. Stepanov, E. Dubois, J. Mag. Mag. Mat. 186 (1998) 175

[22] J. Fresnais, J.-F. Berret, L. Qi, J.-P. Chapel, J.-C. Castaing, O. Sandre, B. Frka-Petesic, R. Perzynski, J. Oberdisse, F. Cousin, Phys. Rev. E, 78 (2008) 040401

[23] O. Griot, J. A. Kitchener, Trans. Faraday. Soc. 61 (1965) 1026

[24] M. J. McGuire, J. Addai-Mensah, K. E. Bremmell, J. Coll. Inter. Sci 299 (2006) 547-555

[25] S. Chibowski, M. Wisniewska, Coll. Surf. A 208 (2002)

131-145

[26] A. K. Bajpai, S. K. Bajpai, Coll. Surf. A 101 (1995) 21-28

[27] G. Mériguet, F. Cousin, E. Dubois, F. Boué, A. Cebers, B. Farago, R. Perzynski, J. Phys. Chem. B 110 (2006) 4378-438 


\title{
Supporting Information
}

\section{Static and dynamic structural probing of swollen Polyacrylamide ferrogels}

\author{
J.A. Galicia ${ }^{1,2}$, F.Cousin ${ }^{3}$, E. Dubois ${ }^{1}$, O. Sandre ${ }^{1}$, V. Cabuil ${ }^{1}$, R. Perzynski ${ }^{1, *}$ \\ ${ }^{1}$ Université Pierre et Marie Curie - PECSA - UMR 7195 CNRS - UPMC - ESPCI, case 51, 4 Place Jussieu, 75252 Paris cedex 05 - France \\ ${ }^{2}$ Fac. de Ing. Quimica, Benemerita Universidad Autonoma de Puebla, Edif 147/102 Ciudad Universitaria,18 sur y San Claudio, Puebla, Pue. Mexico \\ ${ }^{3}$ Laboratoire Léon Brillouin, UMR 12 CNRS-CEA, CE-Saclay, 91191 Gif-sur-Yvette - France
}

* corresponding author,

e-mail : regine.perzynski@upmc.fr

\section{Hydrogels}

\section{1 - Synthesis}

The hydrogels are obtained by a free radicalar polymerization as described in part $2 \mathrm{a}$ of the main article replacing the ferrofluid by an aqueous solution of trisodium citrate $\left(\mathrm{Na}_{3} \mathrm{Cit}\right)$ at a concentration $8.10^{-3} \mathrm{~mol} . \mathrm{L}^{-1}$. The range $(0.5-2 \%)$ of cross-linker ratio is chosen for the following reasons :

(i) Below $\mathrm{CL}=0.5 \%$, the gels are too soft to be manipulated (ii) Above $\mathrm{CL}=2 \%$, PAM hydrogels are known to present local heterogeneities of cross-linkers. ${ }^{1,2,3}$

\subsection{Swelling of the hydrogel}

At the end of the synthesis, the hydrogel is out of thermodynamical equilibrium. Placed inside a water bath at the same concentration $\left[\mathrm{Na}_{3} \mathrm{Cit}\right]$ as the synthesis medium, it absorbs a large volume of water. The swelling equilibrium is reached when the hydrogel mass does not evolve anymore (typically after 3 weeks). Before reaching equilibrium, the bath is changed several times in order to accelerate the equilibration process. When equilibrium is reached, the swollen gel is weighed $\left(\mathrm{m}_{\text {swollen }}\right)$, then dried at $70^{\circ} \mathrm{C}$ during 12 hours, and weighed again $\left(\mathrm{m}_{\text {dried }}\right) . \mathrm{m}_{\text {dried }}$ is very close to the mass of polymer $\mathrm{m}_{\text {polymer }}$ because the polymerization yield is measured equal to $98 \%$. This allows to determine the swelling ratio $G_{H G}^{\text {equil }}$ at equilibrium through $\left(m_{\text {swollen }}-m_{\text {dried }}\right) / m_{\text {polymer }}=m_{\mathrm{H}_{2} \mathrm{O}} / m_{\text {polymer }}$. $G_{H G}^{\text {equil }}$ depends on the degree of cross-linking CL (see table SI.1). It is related to the volume ratio of polymer at equilibrium through $\varphi_{A M}^{\text {equil }}=\rho_{\mathrm{H}_{2} \mathrm{O}} / \rho G_{\mathrm{HG}}^{\text {equil }}$ with $\rho / \rho_{\mathrm{H}_{2} \mathrm{O}}=1.35, \rho$ being the polymer density. The same formula stands at the end of the synthesis : $\varphi_{A M}^{\text {synth }}=\rho_{\mathrm{H}_{2} \mathrm{O}} / \rho G_{H G}^{\text {synth }}$ where $\varphi_{A M}^{\text {synth }}$ and $G_{H G}^{\text {synth }}$ are the volume ratio of polymer and the swelling ratio in the preparation state (after cross-linking and before swelling).

\section{3 -Swelling pressure of hydrogels and mesh size of the hydrogel polymeric array}

Once $G_{H G}^{\text {equil }}$ is measured, it is possible to calculate the initial swelling pressure $\Pi_{\text {swl }}^{H G}$ (at polymer volume fraction $\varphi_{A M}$ $=\varphi_{A M}^{\text {synth }}$ ) following Flory-Rehner theory knowing the Flory parameter $\chi$. It is also possible to calculate the number of monomers $N_{C}$ between two cross-links and to estimate the mesh size of the polymeric network $\xi_{\text {equil }}$ either from the volume density of cross-links or from the gyration radius of a polymer branch between two cross-links, that give very similar results. All the results are summed up in table SI.1 for the three cross-linking ratios CL used here.

\begin{tabular}{|c|c|c|c|c|c|c|c|c|}
\hline $\begin{array}{c}\mathrm{CL} \\
(\%)\end{array}$ & $G_{H G}^{\text {synth }}$ & $\begin{array}{c}\varphi_{A M}^{\text {synth }} \\
(\%)\end{array}$ & $G_{H G}^{\text {equil }}$ & $\begin{array}{c}\varphi_{A M}^{\text {equil }} \\
(\%)\end{array}$ & $\begin{array}{c}N_{c}= \\
M_{c} / M\end{array}$ & $\begin{array}{c}\xi_{\text {equil }} \\
\xi_{\Theta} \\
(\mathrm{nm})\end{array}$ & $\begin{array}{c}\xi_{\text {synth }} \\
(\mathrm{nm})\end{array}$ & $\begin{array}{c}\prod_{\text {swl }}^{H G} \\
(\mathrm{kPa})\end{array}$ \\
\hline 0.5 & 25.9 & 2.78 & 74.4 & 0.99 & $1.5510^{3}$ & $\begin{array}{c}24.0, \\
23.0\end{array}$ & 16.8 & 3.60 \\
\hline 1 & 25.4 & 2.83 & 54.0 & 1.35 & $8.310^{2}$ & $\begin{array}{c}17.5, \\
16.5\end{array}$ & 13.4 & 3.52 \\
\hline 2 & 24.9 & 2.88 & 38.9 & 1.87 & $4.210^{2}$ & 12.5, & 10.8 & 2.93 \\
\hline
\end{tabular}

Table SI.1- Characteristics of the hydrogels - CL cross-linking ratio; $G_{H G}^{\text {synth }}$ and $\varphi_{A M}^{\text {synth }}$ : swelling ratio and polymeric volume ratio of the hydrogel determined immediately after synthesis; $G_{H G}^{\text {equil }}$ and $\varphi_{A M}^{\text {equil }}$ : swelling ratio and polymeric volume ratio at swelling equilibrium; $N_{c}$ is the number of monomers between two cross-links, $M_{c}$ being the molar mass of polymer between cross-links deduced at equilibrium from equ.(SI.4) and $M$ the molar mass of monomer AM; $\xi_{\text {equil }}$ is the mesh size of the hydrogel at swelling equilibrium deduced from equ.(SI.5), $\xi_{\Theta}$ the mesh size of PAM in a $\Theta$ solvent; $\xi_{\text {synth }}$ is the mesh size of the hydrogel immediately after synthesis, $\Pi_{\text {swell }}^{H G}$ the initial swelling pressure of the hydrogel at $\varphi_{A M}=\varphi_{A M}^{\text {synth }}$.

The thermo-dynamical equilibrium of a neutral polymeric array, with a homogeneous distribution of reticulation nodes is ruled by a balance between osmotic and elastic forces. The swelling pressure of the hydrogel $\Pi_{s w l}$ is given by ${ }^{4,5}$ :

$$
\Pi_{s w l}^{H G}=-\frac{1}{V_{1}}\left(\mu_{1}^{g e l}-\mu_{1}^{\text {bath }}\right)=\Pi_{m i x}^{H G}-\Pi_{e l}^{H G}
$$

where $\mu_{1}^{\text {gel }}$ (resp. $\mu_{1}^{\text {bath }}$ ) is the chemical potential of water inside the hydrogel (resp. inside the bath), $V_{1}$ is the molar volume of the aqueous solvent $\left(=18 \mathrm{~cm}^{3} / \mathrm{mol}\right), \Pi_{\text {mix }}^{H G}$ is the contribution coming from entropic and enthalpic effects due to the polymer/solvent mixing, $\Pi_{e l}^{H G}$ is the elastic pressure associated to the stretching of the polymeric chains with respect to their reference molten state (without solvent). We forget here any contribution coming from electrostatic interactions between chains. The mixing pressure writes :

$$
\begin{aligned}
\Pi_{\text {mix }}^{H G} & =-\frac{R_{o} T}{V_{1}}\left(\ln \left(1-\varphi_{A M}\right)+\varphi_{A M}+\chi \varphi_{A M}^{2}\right) \\
& \cong \frac{R_{o} T}{V_{1}}\left(\frac{1}{2}-\chi\right) \varphi_{A M}^{2} \text { if } \varphi_{A M}<<1
\end{aligned}
$$

where $R_{o}$ is the Perfect Gas constant, $T$ the temperature in Kelvin, $\varphi_{\text {AM }}$ the volume fraction of polymer inside the hydrogel and $\chi$ the Flory parameter which is here equal to 0.47 . $^{6,7}$ 
This document is the Accepted Manuscript version of a Published Work that appeared in final form in Soft Matter, 2009, 5, 2614-2624, copyright (C) Royal Society of Chemistry after peer review and technical editing by the publisher. To access the final edited and published work see http://pubs.rsc.org/en/Content/ArticleLanding/2009/SM/b819189a

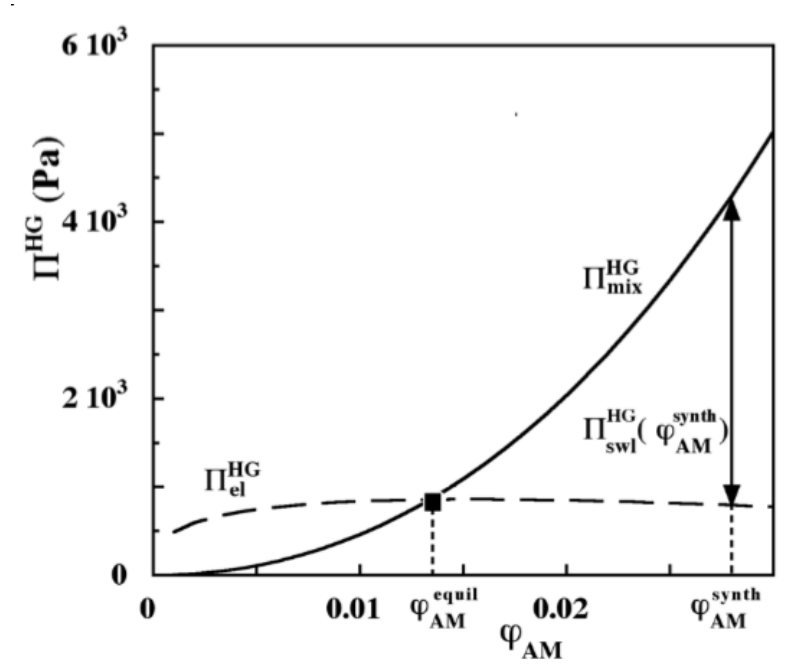

Figure SI.1 - Various pressures of hydrogel and elastic modulus Mixing pressure $\Pi_{\text {mix }}^{H G}$ (equ.(SI.2) - full line) and elastic pressure $\Pi_{e l}^{H G}$ (equ.(SI.3) - dashed line) as a function of the polymer concentration $\varphi_{\mathrm{AM}}$. In the synthesis conditions, $\varphi_{\mathrm{AM}}=\varphi_{A M}^{\text {synth }}$ and the swelling pressure $\Pi_{\text {swell }}^{H G}=\Pi_{\text {mix }}^{H G}-\Pi_{e l}^{H G}$. At swelling equilibrium, $\varphi_{\mathrm{AM}}$ $=\varphi_{A M}^{\text {equil }}$ and $\Pi_{\text {mix }}^{H G}=\Pi_{e l}^{H G}$ (solid square). It is proportional to the elastic modulus of the swollen hydrogel at equilibrium. A coefficient 0.67 is found while comparing to the measurement at $\mathrm{CL}=1 \%$ of ref $^{8}$.

The elastic contribution is related to the entropy of the stretched chains. Supposing an isotropic swelling with an homogeneous distribution of nodes in the hydrogel, we obtain :

$$
\Pi_{e l}^{H G}=R_{o} T v_{c}\left[\varphi_{A M}^{\text {synt }}\left(\frac{\varphi_{A M}}{\varphi_{A M}^{\text {synth }}}\right)^{1 / 3}-\frac{1}{2} \varphi_{A M}\right]
$$

where $v_{c}=\rho / M_{c}$ is the volumic number of nodes in the dry hydrogel (in mol/vol. of polymer), $M_{c}$ being the molar mass between cross-links.

When the system is out of equilibrium, equation (SI.1) governs the swelling of the hydrogel. Figure SI.1 illustrates the $\varphi_{\mathrm{AM}}$-dependence of $\Pi_{\text {mix }}^{H G}$ (full line) and $\Pi_{e l}^{H G}$ (dashed line) at $\mathrm{CL}=1 \%$. Just after synthesis, $\Pi_{\text {mix }}^{H G}$ given by equation (SI.2) with $\varphi_{A M}=\varphi_{A M}^{\text {synth }}$ is much larger than $\Pi_{e l}^{H G}\left(\varphi_{A M}^{\text {synth }}\right) \square \mathrm{s}$ it corresponds to a deformation still small with respect to the melt. In that case, because $\Pi_{s w l}^{H G}$ is positive, the hydrogel absorbs water from the swelling bath. As the hydrogel swells, $\varphi_{\text {AM }}$ decreases, $\Pi_{\text {mix }}^{H G}$ decreases and $\Pi_{e l}^{H G}$ slightly increases, the hydrogel swells less and less. When $\Pi_{s w l}^{H G}=0$, that is when $\Pi_{\text {mix }}^{H G}=\Pi_{e l}^{H G}$, the equilibrium is reached, $\varphi_{A M}=\varphi_{A M}^{\text {equil }}$ and the swelling stops.

The volume fraction of polymer inside the hydrogel at the end of the synthesis is almost the same for all the samples, the slight variation in table SI.1 resulting from the different CL. The volume ratio $\varphi_{A M}^{\text {equil }}$ is deduced from the measurement of the swelling ratio $G_{H G}^{\text {equil }}$. It allows to obtain $M_{c}$ at equilibrium (see table SI.1) through:

$$
M_{c}=V_{1} \rho \frac{\left[\frac{1}{2} \varphi_{A M}^{\text {equil }}-\varphi_{A M}^{\text {synth }}{ }^{2 / 3} \varphi_{A M}^{\text {equil }} 1 / 3\right.}{\ln \left(1-\varphi_{A M}^{\text {equil }}\right)+\varphi_{A M}^{\text {equil }}+\chi \varphi_{A M}^{\text {equil }}{ }^{2}}
$$

We can thus deduce the number of monomers $N_{c}=M_{c} / M$ between two cross-links, $M=71 \mathrm{~g} / \mathrm{mol}$ being the molar mass of monomer AM. We obtain also the mesh size $\xi_{\text {equil }}$ at swelling equilibrium from :

$$
\xi_{\text {equil }}=\left[N_{a} v_{c} \varphi_{A M}^{\text {equil }}\right]^{1 / 3}
$$

Let us note that the state of the system being close to $\Theta$ conditions $(\chi=0.47 \sim 1 / 2)$ the mesh size $\xi_{\text {equil }}$ at swelling equilibrium is closer to $\xi_{\Theta}=a N^{1 / 2}$ than to the Flory mesh size $\xi_{F}=a N^{3 / 5}, \mathrm{a}=0.58 \mathrm{~nm}$ being the length of monomer AM. ${ }^{9,10}$

The experimental evidence that the structure of hydrogels is homogeneous (in the synthesis conditions and at swelling equilibrium) is provided by the fact that the effective concentration of cross-links at swelling equilibrium $\left(\sim 1 / N_{c}\right)$ is found proportional to CL. The mesh size after the synthesis is here given by $\xi_{\text {synth }}=\xi_{\text {equil }}\left(G_{H G}^{\text {synth }} / G_{H G}^{\text {equil }}\right)^{1 / 3}$.

\section{Ferrofluids}

\subsection{Synthesis}

The magnetic nanoparticles are synthesized by coprecipitation in an aqueous ammonia solution of $\mathrm{FeCl}_{2}$ and $\mathrm{FeCl}_{3}$ salts that leads to colloidal magnetite which is fully oxidized to maghemite by $\mathrm{Fe}\left(\mathrm{NO}_{3}\right)_{3}$ in acidic medium. ${ }^{11}$ The experimental conditions have been chosen in order to prepare particles with an average diameter of the order of $10 \mathrm{~nm}$. A size-sorting process allows then a fractionation of the population of nanoparticles according to their diameter. ${ }^{12}$ Finally the initially positively charged nanoparticles are coated with citrate species by addition of tri sodium citrate $\left(\mathrm{Na}_{3} \mathrm{Cit}\right)$ in order to have negative surface charges associated to ionized carboxylate groups for $\mathrm{pH}$ ranging between 6 to 10 . The citrate coated particles yield to stable dispersions in water at $\mathrm{pH} 7$, the electrostatic interparticle repulsion preventing the nanoparticle aggregation. The adsorption equilibrium of citrate gives a residual ionic strength due to unadsorbed (free) citrate species $\mathrm{Cit}^{3-}$ and their $\mathrm{Na}^{+}$counterions. To ensure the citrate coating, one needs to keep free citrate salt concentration $\left[\mathrm{Na}_{3} \mathrm{Cit}\right]$ above a given minimal value, which depends on $\mathrm{pH}^{13}$ At $\mathrm{pH} 7$, the adsorption plateau corresponds to a free citrate concentration inside the solution $\left[\mathrm{Na}_{3} \mathrm{Cit}\right] \geq 2.10^{-3} \mathrm{~mol} \cdot \mathrm{L}^{-1}$.

\subsection{SANS experiments :}

The size and structure of FFA and FFB dispersions have been determined by SANS experiments performed in LLB (Saclay, France) on the PAXY spectrometer, in the wavevector range $0.0065 \AA^{-1} \leq \mathrm{q} \leq 0.15 \AA^{-1}$. In pure light water, the intensity after subtraction of the incoherent background is largely dominated by the nuclear contribution of the particles. ${ }^{14}$ As the nanoparticles are roughly spherical, the intensity $\mathrm{I}(\mathrm{q}, \Phi)$ scattered by a dispersion of volume fraction $\Phi$ writes :

$$
\frac{I(q, \Phi)}{\Phi}=\Delta \rho^{2} F(q) S(q, \Phi)
$$

where $\Delta \rho^{2}=5.6710^{21} \mathrm{~cm}^{-4}$ is the neutronic nuclear contrast of the nanoparticles with respect to $\mathrm{H}_{2} \mathrm{O}, F(q)$ is the form factor of the nanoparticles and $S(q, \Phi)$ the structure factor of the colloidal dispersion. In the dilute regime and if the interparticle interactions are negligible, the structure factor of the dispersions is equal to 1 . The scattered intensity is then proportional to $F(q)$.

Figure SI.2a presents the SANS scattered intensity (divided by $\Phi$ ) for sample FFB at various $\Phi$ and low ionic strength $\left[\mathrm{Na}_{3} \mathrm{Cit}\right]=8.10^{-3} \mathrm{~mol} . \mathrm{L}^{-1}$. The intensity at $\Phi=0$ is proportional to $F(q)$ and gives the size and shape characterization deduced 
This document is the Accepted Manuscript version of a Published Work that appeared in final form in Soft Matter, 2009, 5, 2614-2624, copyright (C) Royal Society of Chemistry after peer review and technical editing by the publisher. To access the final edited and published work see http://pubs.rsc.org/en/Content/ArticleLanding/2009/SM/b819189a

from such an experiment. The plateau at low $\mathrm{q}$ in the Guinier range $\left(\mathrm{q}<2.10^{-2} \AA^{-1}\right)$ obtained at the lowest volume fraction confirms that for $\Phi<1 \%$, the interactions between particles can be almost neglected in the experimental conditions. At large q $\left(\mathrm{q}>6.10^{-2} \AA^{-1}\right)$ in the so-called Porod regime, $\mathrm{I}(\mathrm{q}, \Phi) / \Phi$ roughly decreases as $q^{-4}$ whatever $\Phi$, which is characteristic of solid particles with a sharp interface. Figure SI.2a presents the best fit of the form factor of the FFB particles, assumed spherical with a lognormal distribution of median diameter $d_{O}^{\text {scat }}=7 \mathrm{~nm}$ and polydispersity $\sigma^{\text {scat }}=0.26$ (see table SI. 2 for FFA characteristics).
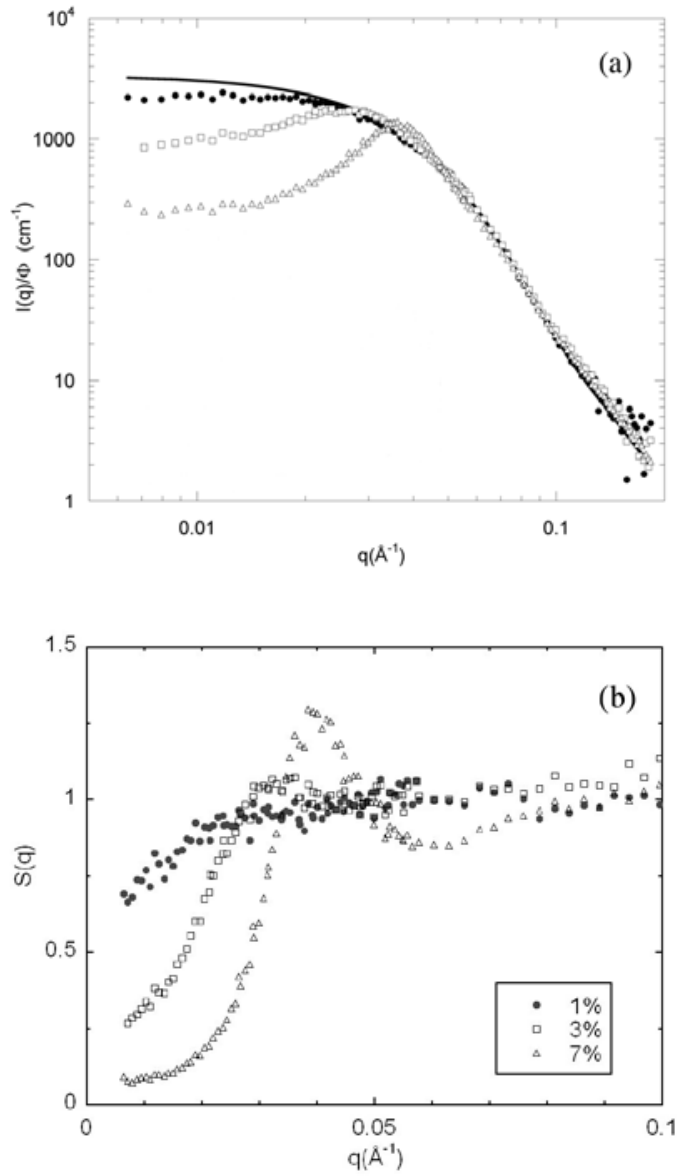

Figure SI.2 SANS probing of FFB ferrofluids at various $\boldsymbol{\Phi}$ - The full lines correspond the form factor of the nanoparticles (see text and table 2). (a) Reduced scattering intensity $\mathrm{I}(\mathrm{q}, \Phi) / \Phi$ versus $\mathrm{q}$ at various $\Phi$; (b) Structure factor of the nanoparticles $\mathrm{S}(\mathrm{q}, \Phi)$ versus $\mathrm{q}$ at various $\Phi$.

For volume fractions $\Phi>1 \%$, the inter-particle interactions are no longer negligible and the structure factor $S(q, \Phi)$ in equation (SI.6) is no more equal to 1. Figure SI 2.b provides the structure factor $S(q, \Phi)$ of three dispersions at volume fractions $\Phi=1 \%, 3 \%$ and $7 \%$, based on the same nanoparticles (FFB) at $\left[\mathrm{Na}_{3} \mathrm{Cit}\right]=8.10^{-3} \mathrm{~mol} . \mathrm{L}^{-1}$. In the thermodynamic limit (for $\mathrm{q} \rightarrow$ 0 ), the structure factor tends to the compressibility of the system. Figure 2 shows that it decreases as the volume fraction increases, which means that the interparticle interaction is repulsive on average. The maximum of $S(q)$ at $q_{\max }$, associated to the most-probable distance $\mathrm{l}_{\mathrm{mp}}=2 \pi / \mathrm{q}_{\max }$ between scattering entities inside the dispersion, increases in amplitude and simultaneously moves towards higher $\mathrm{q}_{\max }$ values. If the interparticle interaction is repulsive on average, the nanoparticles are homogeneously dispersed inside the dispersion. Then the most-probable distance $2 \pi / \mathrm{q}_{\max }$ is also equal to the mean interparticle distance $l_{\text {mean }}$, related to the volume fraction $\Phi$ of the dispersion and the median diameter $\mathrm{d}_{0}$ of particles through $l_{\text {mean }}=d_{o} \sqrt[3]{\frac{\pi}{6 \Phi}}$. For $\Phi=3 \%, l_{\text {mp }}$ is $\sim 196 \AA$ $\left(\mathrm{q}_{\max } \sim 0.032 \AA^{-1}\right)$ and $\mathrm{l}_{\text {mean }}$ is $212 \AA$. For $\Phi=7 \%, \mathrm{l}_{\mathrm{mp}}$ is $\sim 161$
$\AA\left(\mathrm{q}_{\max } \sim 0.039 \AA^{-1}\right)$ and $\mathrm{l}_{\text {mean }}$ is $160 \AA$. The good agreement between those two estimates in our ferrofluid dispersions shows that there are strong repulsive interaction between our nanoparticles at $\left[\mathrm{Na}_{3} \mathrm{Cit}\right]=8.10^{-3} \mathrm{~mol} . \mathrm{L}^{-1}$.

\begin{tabular}{|c|c|c|c|c|c|c|c|c|c|}
\hline & $\begin{array}{c}d_{O}^{\text {scat }} \\
\mathrm{nm}\end{array}$ & $\sigma^{\text {scat }}$ & $\begin{array}{c}d_{O}^{\text {bir }} \\
\mathrm{nm}\end{array}$ & $\sigma^{\text {bir }}$ & $\delta \mathrm{n}_{\mathrm{o}}$ & $\begin{array}{c}\tau_{\mathrm{o}} \\
\mu \mathrm{s}\end{array}$ & $\alpha$ & $\begin{array}{c}\delta \\
\mathrm{nm}\end{array}$ & $\begin{array}{c}\delta \\
\mathrm{nm}\end{array}$ \\
\hline FFA & 9.2 & 0.35 & 11.5 & 0.25 & 0.1 & 5.4 & 0.93 & 12 & 4.5 \\
\hline FFB & 7.0 & 0.26 & 9.1 & 0.2 & 0.07 & 1.8 & 0.95 & 11 & 5 \\
\hline
\end{tabular}

Table SI.2 - Characteristics of the ferrofluid nanoparticles Parameters deduced by an adjustment to a log-normal distribution of diameters, of SANS ( $d_{O}^{\text {scat }}, \sigma^{\text {scat }}$ ) and static birefringence ( $d_{O}^{\text {bir }}, \sigma^{\text {bir }}$ ) measurements; $\delta n_{0}$ is the intrinsic anisotropy of the nanoparticles ${ }^{15} ; \tau_{0}$ characteristic relaxation time and $\alpha$ stretched exponent are deduced from the stretched exponential adjustment of birefringence relaxation using equ. (SI.8); d average nanoparticle diameter and $\delta$ electrostatic screening length are deduced from a Carnahan-Starling adjustment of the $\Phi$-dependence of ferrofluid osmotic pressure $\Pi(\Phi)$ as in ${ }^{16}$.

\subsection{Osmotic equilibrium of Ferrofluids}

In the ferrofluid dispersions used here, the magnetic nanoparticles, due to their citrate coating, are in strong repulsive interaction. The osmotic pressure $\Pi^{F F}$ due to the nanoparticle/solvent mixing can be described in terms of repulsive effective Hard-Spheres following the CarnahanStarling formalism ${ }^{16}$ :

$$
\frac{\Pi^{F F} V}{\Phi k T}=\frac{1+\Phi+\Phi^{2}+\Phi^{3}}{(1-\Phi)^{3}}
$$

where $V=\frac{\pi}{6} d^{3}$, d being an averaged nanoparticle diameter. In the limit $\Phi \rightarrow 0$, the right hand term is equal to 1 and equation (SI.7) reduces to the Perfect Gas law, also valid in the limit of low $\Phi$ if the balance of interparticle interaction is nul (i.e. if the second virial of the osmotic pressure equals zero). Here the balance is repulsive and the repulsions are taken into account in the right hand term of equation (SI.7) replacing $\Phi$ by an effective volume fraction $\Phi_{\text {eff }}$, which includes the range of the repulsion. ${ }^{16}$ Indeed the repulsion is strong enough to consider the nanoparticles as Hard-Spheres with an effective diameter $\mathrm{d}$ $+2 \delta$, where $\delta$ is the electrostatic screening length. It leads to an effective volume fraction $\Phi_{\text {eff }}=\Phi(1+2 \delta / d)^{3}$. Figure 2 of the main text presents such adjustments for the two ferrofluids used here. We obtain $\mathrm{d}=12 \mathrm{~nm}$ and $\delta=4.5 \mathrm{~nm}$ for FFA (resp. $\mathrm{d}=11 \mathrm{~nm}$ and $\delta=5 \mathrm{~nm}$ for FFB).

\section{4 - Magnetic and magneto-optical properties}

Each nanoparticle bears a magnetic moment $\frac{\mathrm{L}}{\sqrt{t}}$ which is of the order of $10^{4}$ Bohr magnetons and an optical anisotropy axis 䒘. Its modulus $\mid=m_{S} V_{\text {magn }}$ is proportional to the magnetic volume $\mathrm{V}_{\text {magn }}$ of the nanoparticles and to $\mathrm{m}_{\mathrm{S}}$ the magnetization of the nanoparticles material. Under a large applied field

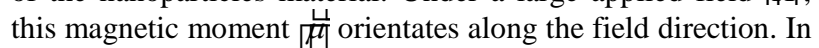
a liquid dispersion of $10 \mathrm{~nm}$-sized $\gamma-\mathrm{Fe}_{2} \mathrm{O}_{3}$ nanoparticles, the magnetic torque also rotates mechanically the core of the nanoparticle together with its optical axis. At equilibrium in large fields, the optical axis $1 \vec{\phi}$ becomes parallel to alignment of $\frac{\dot{m}}{\sqrt{t}}$ along $\frac{\mathrm{H}}{\mathrm{H}}$ confers to the dispersion a macroscopic magnetization $\mathrm{M}$. The alignment of the optical axis $\ddot{\mathbb{\phi}}$ of all the nanoparticles gives a macroscopic optical birefringence $\Delta \mathrm{n}$ to the dispersion. At saturation, all the 
This document is the Accepted Manuscript version of a Published Work that appeared in final form in Soft Matter, 2009, 5,

2614-2624, copyright (C) Royal Society of Chemistry after peer review and technical editing by the publisher. To access the final edited and published work see http:// pubs.rsc.org/en/Content/ArticleLanding/2009/SM/b819189a

magnetic moments and all the optical axes are aligned along

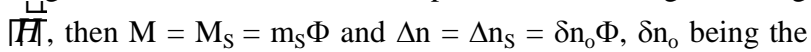
intrinsic optical anisotropy parameter of the nanoparticles. ${ }^{17}$ For intermediate fields and low $\Phi$ 's, $\mathrm{M}$ and $\Delta \mathrm{n}$ are well described by a Langevin formalism with in particular $\Delta \mathrm{n}=$ $\Delta \mathrm{n}_{\mathrm{S}} \mathrm{L}_{2}(\zeta)$, where $\mathrm{L}_{2}(\zeta)=1-3 \mathrm{~L}(\zeta) / \zeta, \quad \mathrm{L}(\zeta)=\operatorname{ctanh}(\zeta)-1 / \zeta$ and $\zeta=\frac{\mu_{0} \mu H}{k T}$ being respectively the Langevin function and the Langevin parameter. However, the shape of $\Delta \mathrm{n}(\mathrm{H})$ is modified by the size distribution of the nanoparticles. ${ }^{17}$ In ferrofluids at low $\Phi$ 's, the experimental measurements of magneto-optical birefringence as a function of the applied field enable the determination of ( $d_{O}^{\text {bir }}, \sigma^{\text {bir }}$ ) by comparison to the theoretical Langevin formalism taking into account a log-normal sizedistribution (see table SI.2 and best fit of $\Delta \mathrm{n}(\mathrm{H})$ for FFA in fig.6).

The magneto-optical birefringence $\Delta \mathrm{n}(\mathrm{H})$ is measured as in ${ }^{15}$ up to a few $100 \mathrm{kA} / \mathrm{m}$. Figure 6 of main text presents the variations of $\Delta \mathrm{n}(\mathrm{H})$ normalized by its volume fraction $\Phi$ for a sample based on FFA nanoparticles at $\left[\mathrm{Na}_{3} \mathrm{Cit}\right]=8.10^{-3} \mathrm{~mol} . \mathrm{L}^{-}$ ${ }^{1}$ for $\Phi=1 \%$ and the corresponding fit. The maximum value $\Delta \mathrm{n}_{\mathrm{S}}$ of $\Delta \mathrm{n}$ is proportional to the volume fraction $\Phi$, the proportionality coefficient $\delta n_{0}$ being dependent on the nanoparticle size distribution. ${ }^{15}$ The values of $\delta n_{0}$ for FFA and FFB are given in Table SI.2. Figure 6 of main text presents also the reduced variations of $\Delta \mathrm{n}(\mathrm{H}) / \Delta \mathrm{n}_{\mathrm{S}}$ at $\Phi=7 \%$. The shape of the curve is almost independent on $\Phi$ in our range of volume fractions expressing the weak influence of interparticle interactions on magneto-optical birefringence in the present repulsive regime.

The ferrofluid solutions can be also probed from a dynamic point of view. Using the same experimental device as the one described in ${ }^{18}$, we analyze the optical rotational answer of the colloid to a pulse of magnetic field of small amplitude $(\mathrm{H}=4.8$ $\mathrm{kA} / \mathrm{m}$ ) and duration ( 100 ms). The magnetic particles, being suspended in a liquid, align along the field direction during the pulse. It leads to a birefringence which, in low-field approximation, writes $\Delta n_{L F}=\delta n_{O} \Phi \zeta^{2} / 15$. Here $\zeta \leq 0.14$ and $\Delta \mathrm{n}_{\mathrm{LF}}$ is at most of the order of $10^{-5}$. The temporal analysis of the birefringence relaxation at the cut-off of the field is performed in terms of a stretched exponential decay of optical intensity $I(t)$ (see figure SI.3a):

$$
I(t)=I_{o} e^{-\left(\frac{\tau}{\tau_{o}}\right)^{\alpha}}
$$

with $I(t) \propto \sin \varphi, \varphi$ being the (here time-dependent) phase-lag of the birefringent sample. We can determine three physical quantities : the initial intensity $I_{o}$, the characteristic relaxation time $\tau_{\mathrm{o}}$ and the stretched exponent $\alpha$. Providing that the phaselag $\varphi$ of the birefringent sample is small enough, the intensity $\mathrm{I}_{\mathrm{o}}$ is proportional to $\Delta \mathrm{n}_{\mathrm{LF}}$. The condition $\varphi=2 \pi \mathrm{e} \Delta \mathrm{n}_{\mathrm{LF}} / \lambda<<1$, where e is the thickness of the optical sample cell (here of the order of $100 \mu \mathrm{m}$ ) and $\lambda$ the optical wave-length (here $=0.633$ $\mu \mathrm{m})$, is easily fulfilled ( $\varphi \leq 10^{-2}$ ). Thus we obtain in the liquid suspension:

- for the intensity $I_{0}$ of the magneto-optical pulse

$$
I_{o} \propto \Phi \zeta^{2} \propto \Phi V_{\text {magn }}^{2}
$$

- for the time $\tau_{0}$, which is proportional to the characteristic time of rotational diffusion:

$$
\tau_{o}=\frac{\eta V_{H}}{k T}
$$

with $\eta$ the viscosity of the liquid carrier and $V_{H}$ the hydrodynamic volume of the nanoparticles.

- for the exponent $\alpha$, which is related to the width of the distribution of relaxation times in the dispersion

$$
\alpha \approx 1
$$

for samples based on nanoparticles of thin distribution of sizes. This $\alpha$ value is decreasing towards 0.6 in polydisperse or agglomerated liquid dispersions ${ }^{18,19}$ and may reach a value of 0.3 in arrested thixotropic gels ${ }^{18}$ or glass-forming systems ${ }^{19}$.

The stretched-exponential nature of the relaxation of the magneto-optical signal is illustrated in the inset of figure SI.3a for sample FFA at $\Phi=3 \%$ and $\left[\mathrm{Na}_{3} \mathrm{Cit}\right]=8.10^{-3} \mathrm{~mol} \cdot \mathrm{L}^{-1}$. The almost linear variation of $-\ln \left(\mathrm{I} / \mathrm{I}_{0}\right)$ as a function of time $t$ in this log-log representation, shows that there is only one stretched exponential relaxation process involved in the decay of intensity. The adjusted straight line crosses the horizontal axis - $\ln (\mathrm{I} / \mathrm{Io})=1$ at $\mathrm{t}=\tau_{\mathrm{o}}=5.4 \mu \mathrm{s}$. It has a slope $\alpha=0.93$. In our range of $\Phi$ and within our experimental accuracy, this relaxation time is found here independent of $\Phi$. Table SI.2 gives $\tau_{\mathrm{o}}$ (denoted $\tau_{o}^{F F}$ ) and $\alpha$, obtained for the two kinds of samples based on FFA and FFB nanoparticles.
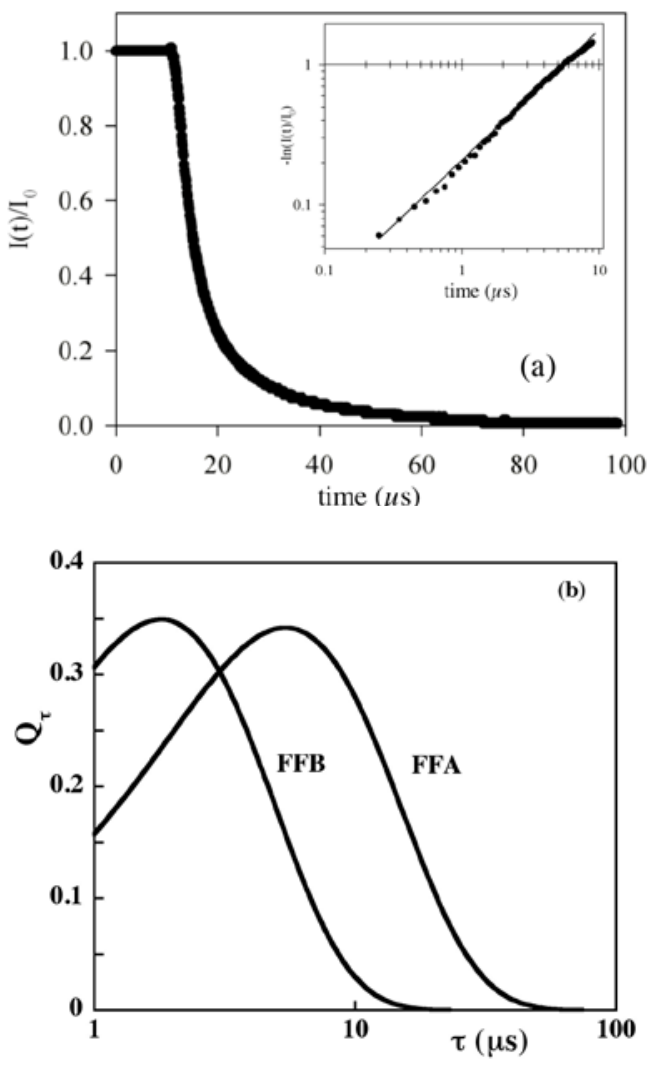

Figure SI.3 - Dynamic birefringence of ferrofluids

$\boldsymbol{a}$ - Relaxation of the optical intensity due to the birefringence of the dispersion under applied magnetic field (FFA with [cit] $=810^{-3} \mathrm{~mol} / \mathrm{L}$ and $\Phi=3 \%$ ). The inset illustrates that the decay is a stretched exponential by plotting $-\ln \left(/ / \mathrm{I}_{0}\right)$ as a function of time $t$ in a $\log -\log$ representation.

$\boldsymbol{b}$ - Distribution of characteristic relaxation times for FFA and FFB ferrofluids as obtained from Equ. SI.13.

In table SI.2, the values of $\alpha$ are rather close to 1 but different from 1, it expresses that the relaxation is non-exponential. Such a non-exponential relaxation is usually asserted to the existence 
This document is the Accepted Manuscript version of a Published Work that appeared in final form in Soft Matter, 2009, 5, 2614-2624, copyright (C) Royal Society of Chemistry after peer review and technical editing by the publisher. To access the final edited and published work see http:// pubs.rsc.org/en/Content/ArticleLanding/2009/SM/b819189a

of a distribution of times $\tau$ inside the ferrofluid. In these liquid solutions it is associated to the polydispersity in hydrodynamic size of the nanoparticles. This temporal relaxation can be inverted like in ${ }^{20}$. It leads to a distribution $Q(\ln (\tau))$ of relaxation times $\tau$ such that :

$$
I(t)=\int I_{o}(\tau) e^{-\frac{t}{\tau}} Q(\ln (\tau)) d(\ln (\tau))
$$

Providing the approximations $I_{o}(\tau) \approx I_{o} \approx$ cst for a given sample, $e^{-t / \tau}=1$ for $\mathrm{t}<\tau$ and $e^{-t / \tau}=0$ for $\mathrm{t}>\tau$, it comes ${ }^{19}$ :

$$
Q(\ln \tau)=-\frac{d\left(I(t) / I_{o}\right)}{d(\ln t)}=\alpha\left(\frac{\tau}{\tau_{o}}\right)^{\alpha} e^{-\left(\frac{\tau}{\tau_{o}}\right)^{\alpha}}
$$

Figure SI3.b plots these distributions of characteristic times (denoted $\mathrm{Q}_{\tau}$ all along this text) for the two kinds of nanoparticles employed here FFA and FFB. Note that in our experimental range of $\Phi$ and $\left[\mathrm{Na}_{3} \mathrm{Cit}\right]$ the obtained distributions are independent of $\Phi$ and $\left[\mathrm{Na}_{3} \mathrm{Cit}\right]$.

\section{3 - Rotational diffusion of nanoparticles within ferrogels probed by dynamical birefringence}

Such a dynamical probing may be also performed in ferrogels. Here below the index "FG" stands either for "synth" or "equil" depending on the state of the ferrogel either in the synthesis conditions or at swelling equilibrium.
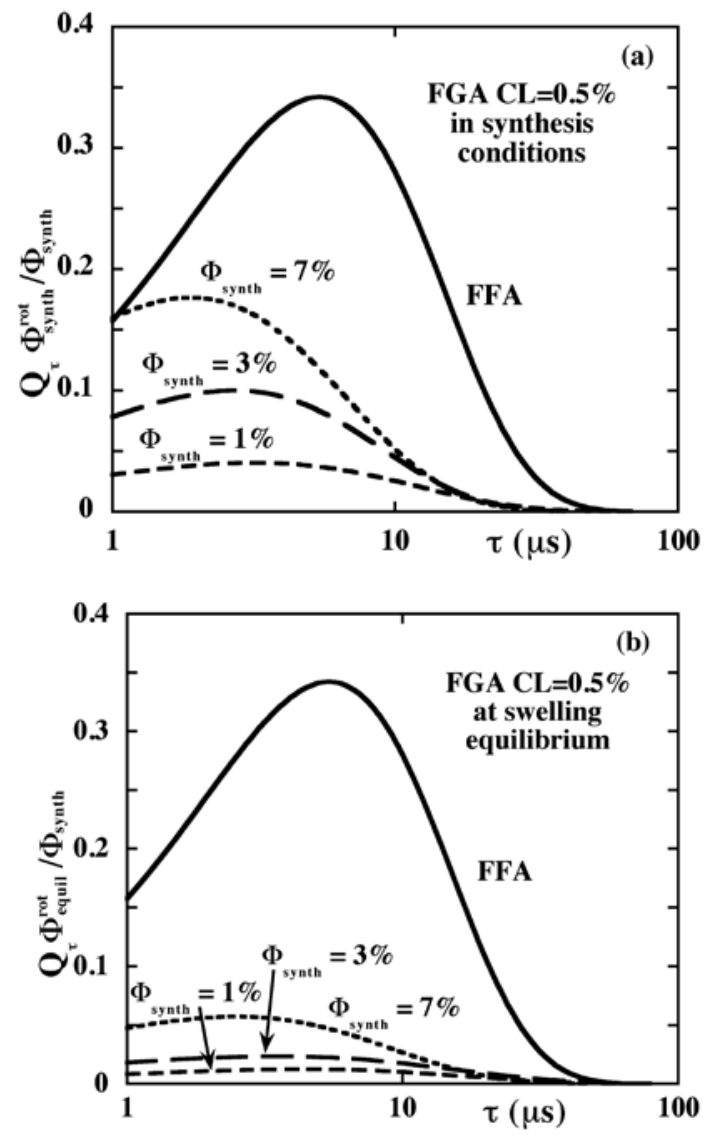

Figure SI.4 - Dynamic birefringence ferrogels FGA - CL $=0.5 \%-$ Reduced distribution of characteristic times $\mathrm{Q}_{\tau} \Phi_{F G}^{\text {rot }} / \Phi_{\text {synth }}$ inside ferrogels at various $\Phi_{\text {synth }}$, in the synthesis conditions (a) and at swelling equilibrium (b) as compared to pure ferrofluid FFA.

Performing two experiments, one with a ferrogel and one with its corresponding ferrofluid, at the same volume fraction $\Phi_{\mathrm{FF}}=\Phi_{\mathrm{FG}}$ and with the same sample thickness $e$, it is also possible to compare the respective initial intensities $I_{o}^{F G}$ and $I_{o}^{F F} . I_{o}^{F G}$ is here much lower than $I_{o}^{F F}$ and at most equal to $I_{o}^{F F} / 10$. Using equations (SI.10) and (SI.11), and with the hypothesis that the ratio of magnetic volumes is proportional to the ratio of hydrodynamic volumes, we obtain :

$$
\frac{I_{o}^{F G}}{I_{o}^{F F}}=\frac{\Phi_{F G}^{r o t}}{\Phi_{F F}}\left(\frac{\tau_{o}^{F G}}{\tau_{o}^{F F}}\right)^{2}
$$

Here $\Phi_{F G}^{r o t}$ is the volume fraction of rotating nanoparticles inside the ferrogel in the low-field experiment of birefringence relaxation. The ratios $I_{o}^{F G} / I_{o}^{F F}$ and $\tau_{o}^{F G} / \tau_{o}^{F F}$ being both measured, it is possible using equation (SI.14) to deduce $\Phi_{F G}^{\text {rot }} / \Phi_{F G}$ the proportion (in volume) of nanoparticles rotating inside the ferrogel in this low field experiment. It is also possible to calculate the volume fraction $\Phi_{F G}^{\text {block }}$ of nanoparticles blocked in low field using $\Phi_{F G}^{\text {block }}=\Phi_{\mathrm{FG}}-\Phi_{F G}^{\text {rot }}$. They are given in tables 2, 3 and 5 of main text for the various samples probed here. Figure SI.4 illustrates for FGA ferrogels at CL $=0.5 \%$, the reduced distributions of characteristic times $\mathrm{Q}_{\tau} \Phi_{\text {synth }}^{\text {rot }} / \Phi_{\text {synth }}$ obtained in non swollen samples at various $\Phi_{\text {synth }}$ (see Figure SI.4a) and the corresponding distributions $\mathrm{Q}_{\tau} \Phi_{\text {equil }}^{\text {rot }} / \Phi_{\text {synth }}$ obtained in swollen samples at various $\Phi_{\text {synth }}$ (see Figure SI.4b). In these figures, the factors $\Phi_{\text {synth }}^{\text {rot }} / \Phi_{\text {synth }}$ and $\Phi_{\text {equil }}^{\text {rot }} / \Phi_{\text {synth }}$ allow a quantitative visualization of the nanoparticles which are rotating in the ferrogel with respect to those initially introduced.

\section{References}

[1] L. Benguigui, F. Boué, Eur. Phys. J. B 11 (1999) 439

[2] D. Asnaghi, M. Giglio, A. Bossi, P.G. Righetti Macromolecules 30 (1997) 6194

[3] Xiaomi Zhang, Zhibing Hu, Yong Li, Polymer 39 (1998) 2783

[4] J. Bastide, S. Candau, L. Leibler, Macromolecules 14 (1981) 719

[5] A. Hochberg, T. Tanaka, D. Nicoli, Phys. Rev. Lett. 43 (1979) 217

[6] T. Hino, J.M. Prausnitz, J. Appl. Polym. Sci. 62 (1996) 1635-1640

[7] P.J. Flory, Principle of Polymer chemistry (Cornell University Press, Ithaca - NY, 1953)

[8] J.A. Galicia, O. Sandre, F. Cousin, D. Guemghar, C. Ménager, V. Cabuil, J. Phys.: Condens. Matter 15 (2003) S1379

[9] Polymer Data Handbook (Oxford University Press, 1999)

[10] Schwartz, T., J. Sabbadin, and J. François, Polymer 22 (1981) 609

[11] R. Massart, I.E.E.E. Trans. Magn. 17 (1981) 1247

[12] R. Massart, E. Dubois, V. Cabuil, E. Hasmonay, J. Magn. Magn. Mat. 149 (1995) 1

[13] E. Dubois, V. Cabuil, F. Boué, R. Perzynski, J. Chem. Phys. 111 (1999) 7147

[14] F. Gazeau, F. Boué, E. Dubois, R. Perzynski, J. Phys.: Condens. Matter 15 (2003) S1305

[15] E. Hasmonay, E. Dubois, J.-C. Bacri, R. Perzynski, Yu. L. Raikher, V.I. Stepanov, Eur. Phys. J. B 5 (1998) 859

[16] F. Cousin, E. Dubois, V. Cabuil, Phys. Rev. E 68 (2003) 021405

[17] Magnetic Fluids and Applications Handbook, ed. by B. Berkovski (Begell House Inc. Publ., New York, 1996)

[18] E. Hasmonay, A. Bee, J.-C. Bacri, R. Perzynski, J Phys Chem B 103 (1999) 6421-6428

[19] G. Mériguet, E. Dubois, V. Dupuis, R. Perzynski, J. Phys.: Condens. Matter 18 (2006) 10119 
This document is the Accepted Manuscript version of a Published Work that appeared in final form in Soft Matter, 2009, 5, 2614-2624, copyright @ Royal Society of Chemistry after peer review and technical editing by the publisher. To access the final edited and published work see http:// pubs.rsc.org/en/Content/ArticleLanding/2009/SM/b819189a

[20] C. Wilhelm, F. Gazeau, J. Roger, J.N. Pons, M.F. Salis, R.

Perzynski, J.-C. Bacri, Phys. Rev. E 65 (2002) 031404 